\title{
Local Anesthetics as Effectors of Allosteric Gating \\ Lidocaine Effects on Inactivation-deficient Rat Skeletal Muscle Na Channels
}

\author{
Jeffrey R. Balser, ${ }^{\ddagger}$ H. Bradley Nuss, ${ }^{*}$ David W. Orias, ${ }^{\star}$ David C. Johns, ${ }^{*}$ Eduardo Marban, ${ }^{\star}$ Gordon F. Tomaselli, \\ and John H. Lawrence* \\ *Department of Medicine and ${ }^{\ddagger}$ Department of Anesthesiology and Critical Care Medicine, The Johns Hopkins University School of \\ Medicine, Baltimore, Maryland 21205
}

\begin{abstract}
Time- and voltage-dependent local anesthetic effects on sodium (Na) currents are generally interpreted using modulated receptor models that require formation of drug-associated nonconducting states with high affinity for the inactivated channel. The availability of inactivation-deficient $\mathrm{Na}$ channels has enabled us to test this traditional view of the drug-channel interaction. Rat skeletal muscle Na channels were mutated in the III-IV linker to disable fast inactivation (F1304Q: FQ). Lidocaine accelerated the decay of whole-cell FQ currents in Xenopus oocytes, reestablishing the wildtype phenotype; peak inward current at $-20 \mathrm{mV}$ was blocked with an $\mathrm{IC}_{50}$ of $513 \mu \mathrm{M}$, while plateau current was blocked with an $\mathrm{IC}_{50}$ of only $74 \mu \mathrm{M}(P<0.005$ vs. peak). In single-channel experiments, mean open time was unaltered and unitary current was only reduced at higher drug concentrations, suggesting that open-channel block does not explain the effect of lidocaine on FQ plateau current. We considered a simple model in which lidocaine reduced the free energy for inactivation, causing altered coupling between activation and inactivation. This model readily simulated macroscopic $\mathrm{Na}$ current kinetics over a range of lidocaine concentrations. Traditional modulated receptor models which did not modify coupling between gating processes could not reproduce the effects of lidocaine with rate constants constrained by single-channel data. Our results support a reinterpretation of local anesthetic action whereby lidocaine functions as an allosteric effector to enhance $\mathrm{Na}$ channel inactivation. (J. Clin. Invest. 1996. 98:2874-2886.) Key words: lidocaine $\bullet$ modulated receptor • single-channel recording • site-directed mutagenesis $\bullet \mathrm{Na}$ channels
\end{abstract}

\section{Introduction}

Sodium $(\mathrm{Na})$ channels are membrane-spanning proteins that form the basis of excitability in neuronal tissue, skeletal muscle, and cardiac muscle. These dynamic macromolecules are

Address correspondence to Jeffrey R. Balser, Department of Anesthesiology and Critical Care Medicine, The Johns Hopkins University School of Medicine, Carnegie 442, 600 N. Wolfe St., Baltimore, MD 21287. Phone: 410-614-0401; FAX: 410-955-7953; E-mail: jrbalser @welchlink.wlech.jhu.edu

Received for publication 10 June 1996 and accepted in revised form 9 October 1996.

J. Clin. Invest.

(c) The American Society for Clinical Investigation, Inc. 0021-9738/96/12/2874/13 \$2.00

Volume 98, Number 12, December 1996, 2874-2886 exquisitely sensitive to the voltage across the cell membrane. When initially depolarized, Na channels assume an open configuration through a gating process referred to as activation. During maintained depolarization, $\mathrm{Na}$ channels eventually assume a nonconducting state through a distinct process known as inactivation. Inactivated channels cannot reopen; the channels must first recover to the resting conformation by spending a repriming period at a hyperpolarized membrane potential. Thus, when channels are subjected to a train of depolarizing pulses in rapid succession, the availability of channels to open declines progressively as they accumulate in the inactivated state, a phenomenon referred to as use dependence. Local anesthetics such as lidocaine bind to and block Na channels in a complex time-dependent manner. A major functional effect of local anesthetics is to enhance use dependence and thereby to suppress cellular excitability (1), particularly at high rates of stimulation.

It is still unclear how local anesthetics exert their complex actions on Na channels. The effects have generally been interpreted using modulated receptor theory $(2,3)$, which postulates that local anesthetics bind preferentially to the inactivated state of the channel. High-affinity local anesthetic binding induces additional nonconducting states which further reduce the probability of channel opening. Although mathematical models based on the modulated receptor hypothesis have successfully reproduced use-dependent local anesthetic effects, firm conclusions are necessarily limited by the assumptions required to choose a model for Na channel gating. Furthermore, while the majority of lidocaine block appears to develop gradually during depolarization with a time course parallel to channel inactivation, a significant fraction of local anesthetic block also develops very rapidly, suggesting an additional interaction between the local anesthetic and the open channel (4). It therefore remains uncertain whether the functionally relevant local anesthetic mechanism in use dependence is plugging of the open pore by the cationic local anesthetic species (5-8) or direct binding to (and stabilization of) the inactivated state (3, $9,10)$. Either mechanism could enhance use dependence if the drug-bound state liberates drug slowly enough to delay channel repriming.

Cloning and expression of the Na channel have enabled detailed structure-function studies aimed at discerning the molecular basis of use dependence. In this regard, a recent study has shown that mutation of a pore-lining phenylalanine residue markedly reduces use-dependent block of Na channels by etidocaine (11). Nevertheless, there is reason to question whether block of the open pore has functional relevance. In early studies, use-dependent block of $\mathrm{Na}$ channels by a permanently charged lidocaine derivative (QX-314) was largely eliminated when channel inactivation was removed enzymatically (6). Gating current measurements support the view that inactivation and QX-314 block are functionally linked (12). In the 
human cardiac Na channel, lidocaine-induced use dependence is reduced when fast inactivation is eliminated by mutation of a triplet of hydrophobic III-IV linker residues $(13,14)$. Experiments with long-chain alkylammonium compounds further suggest that local anesthetic molecules may interact directly with the inactivation mechanism (15).

To clarify the nature of the interaction between local anesthetics and the inactivation gating mechanism, we utilized sitedirected mutagenesis to disable, but not eliminate, Na channel fast inactivation. We replaced a single phenylalanine residue in the III-IV linker of the rat skeletal muscle Na channel $(\mu 1)^{1}$ with glutamine (F1304Q) (13) and evaluated the effect of lidocaine on these inactivation-disabled channels when expressed in Xenopus oocytes. In contrast to earlier reports with fast inactivation entirely eliminated $(6,14)$, lidocaine enhanced the decay of current flowing through F1304Q channels, essentially restoring the wild-type phenotype. We implemented a contemporary scheme for $\mathrm{Na}$ channel gating that incorporates allosteric coupling between activation and fast inactivation (16). When lidocaine effects were modeled as a simple dosedependent reduction in the free energy for inactivation, the allosteric coupling between activation and inactivation was modified, and our experimental results were faithfully reproduced. In contrast, traditional open and inactivated-state modulated receptor models that did not modify coupling between gating processes were incapable of simulating the effects of lidocaine within the kinetic constraints imposed by the single-channel data. These findings necessitate a fundamental revision of the prevailing concepts regarding the mechanism of local anesthetic action.

\section{Methods}

Mutagenesis and Na channel expression. Site-directed mutagenesis of residues in the III-IV linker region of the rat skeletal muscle Na channel $\alpha$ subunit (17) was performed as previously described $(18,19)$. A 2.5-kb Sph 1-Kpn 1 restriction digest fragment from the $\mu 1 \mathrm{cDNA}$ (nucleotides 2230 to 4760) was subcloned into pGEM-7Zf(+) (Promega Corp., Madison, WI). Using this cassette, either the 1304position phenylalanine (F), or the entire 1303-1305 isoleucine, phenylalanine, methionine (IFM) triplet was replaced with glutamines (Q) (13) using oligonucleotide-directed mutagenesis and phenotypic selection (20). After sequence confirmation, the resulting F1304Q (FQ) or I1303Q-F1304Q-M1305Q (QQQ) mutant cassettes were subcloned back into the full-length $\mu 1$ in the oocyte expression vector pSP64T (21), and the entire plasmid was once again confirmed by dideoxy-DNA sequencing in the III-IV linker region (22). The $\alpha$ subunits were expressed by cRNA microinjection into Xenopus oocytes. As described in the results, in selected experiments cRNA encoding the auxiliary $\beta_{1}$ subunit (23) was coinjected with the $\alpha$ subunits in a 1: 1 molar ratio to augment channel expression (24). For FQ experiments in HEK-293 cells, a plasmid was constructed with a cassette containing an RSV promoter, the mutant Na channel coding sequence, and a bovine growth hormone polyadenylation signal. This cassette was cloned into the $\cos 203$ vector (25), and was maintained as an episome in cells selected with hygromycin B.

Electrophysiology. Whole-cell currents in Xenopus oocytes were measured using a two-electrode voltage clamp (Warner, Hamden, $\mathrm{CT}$ ). The pipette contained $3 \mathrm{M} \mathrm{KCl}$ and the bath solution was ND-96

1. Abbreviations used in this paper: $\mu 1$, rat skeletal muscle Na channel; FQ, F1304 mutation; QQQ, I1303-F1304-M1304Q triple mutation. frog Ringers solution containing (mM): $96 \mathrm{NaCl}, 2 \mathrm{KCl}, 1 \mathrm{MgCl}_{2}, 5$ Hepes, $\mathrm{pH}$ 7.6. Lidocaine-HCl (Abbott Laboratories, North Chicago, IL) was added in appropriate amounts to the bath solution to collect paired dose-response data. Full equilibration of the drug in the bath and the oocyte was monitored by applying infrequent depolarizing pulses during the wash-in period. We find that $3-5 \mathrm{~min}$ of perfusion is necessary to provide steady state lidocaine effects on peak Na current. Voltage clamp protocols are described in the figure legends. For dose-response measurements, oocytes were held at $-100 \mathrm{mV}$ for at least $30 \mathrm{~s}$ before each test depolarization. Currents were sampled at $10 \mathrm{kHz}$ and filtered at $1 \mathrm{kHz}$. For noninactivating currents (FQ, QQQ), leak was corrected using an assumption of linearity by subtracting $20 \%$ of the holding current at $-100 \mathrm{mV}$ from the entire macroscopic current recorded at $-20 \mathrm{mV}$. Dose-response data from individual oocytes were fitted to a logistic function $\left(1 /\left(1+[\mathrm{D}] / \mathrm{IC}_{50}\right)\right)$, and differences between the fitted $\mathrm{IC}_{50}$ values for wild-type and mutant $\mathrm{Na}$ channels were compared using one-way ANOVA (MicroCal Origin, Northampton, MA). Only dose-response experiments with control data and two or more concentrations of drug were included in the analysis.

The effects of lidocaine on single-channel currents from wild-type and FQ channels were studied in cell-attached patches (26). The patch pipettes contained $(\mathrm{mM}): 140 \mathrm{NaCl}, 10$ Hepes, $1 \mathrm{BaCl}_{2}(\mathrm{pH}$ 7.4), and the bath solution was nominally Na-free and contained 140 $\mathrm{KCl}, 10$ Hepes, $\mathrm{pH}$ 7.4. Lidocaine effects were studied unpaired by addition of drug to the pipette solution at a $300 \mu \mathrm{M}$ concentration. A 5-min equilibration period after patch formation was allowed to establish steady state effects of lidocaine on the channel in the patch. There were no time-dependent lidocaine effects on mean open time or on the probability of late reopenings during experimental periods lasting up to $60 \mathrm{~min}$. To examine the effects of QX-314 ( $N$-(2,6-dimethylphenyl-carbamoylmethyl) triethylammonium bromide; Alomone Labs, Jerusalem, Israel) on FQ channels, it was necessary to use excised inside-out patches with QX-314 added to the perfusate. The pipette and bath solutions were otherwise the same as for the cellattached patch experiments with lidocaine and FQ described above. To increase the likelihood of recording $\mathrm{Na}$ channels in excised patches, we coexpressed the FQ $\alpha$ subunit with $\beta_{1}$. Such coexpression increases the number of functional Na channels in the Xenopus membrane without altering the permeation properties (27). Pipettes were polished to 4-7 megaohms and tips were coated with Sylgard. All experiments were performed at $20-22^{\circ} \mathrm{C}$.

Single-channel data were collected at a sampling rate of $10 \mathrm{kHz}$ and filtered at $2 \mathrm{kHz}$ using an Axopatch 200A patch clamp amplifier and a TL1 DMA interface (Axon Instruments, Burlingame, CA). Data were stored on hard disk for off-line analysis using custom-written software in Microsoft Visual Basic ${ }^{\mathrm{TM}}$ for Windows ${ }^{\mathrm{TM}}$ (version 2.0). Single-channel records were idealized for open-time analysis using a half-amplitude criterion (28). All data are reported as mean \pm SD unless otherwise noted.

Markov modeling. To examine the effect of lidocaine on FQ gating, we fitted whole-cell currents to a contemporary gating model using a global fitting approach (29-31). The gating model, which utilizes allosteric coupling between activation and inactivation, was recently described by Kuo and Bean (16) for Na channel gating in rat CA1 neurons, and is described in detail in the Results section (Eq. 2). In previous work (31) we used an expanded version of Eq. 2 with two competing, parallel inactivation processes to account for the multiple kinetic components in the FQ ensemble average current decay (see Fig. $4 \mathrm{~B}$ ). However, given our goal of expanding the model to include local anesthetic effects, and recognizing the large number of additional nonconducting states required by modulated receptor models of drug action, we utilized the original formulation of the Kuo and Bean model with only a single parallel inactivation process (Eq. 2, Results). Hence, the decay of simulated drug-free FQ current (see Fig. 8) appears monoexponential, unlike ensemble average currents simulated previously (31). Furthermore, the two-electrode voltage clamp in oocytes does not reproduce the rapid wild-type $\mathrm{Na}$ channel 
activation and inactivation kinetics observed in ensemble-average currents recorded from cell-attached patches. Because we utilized the mean open times from single-channel experiments to restrict the rate constant parameter search, the resulting simulated wild-type currents in Fig. 8 better reflect ensemble average current behavior (see Fig. $4 \mathrm{~B}$ ) than the slower activation and decay kinetics seen in whole oocyte measurements (see Fig. 1). Nonetheless, because the decay of FQ current during depolarization is far slower than wild-type, the model-generated ensemble average FQ current decay compares favorably with that measured in whole-oocyte experiments; hence, the rate constants given in the table in Fig. 8 (bottom) were utilized as fixed parameters in our efforts to model the effects of lidocaine on whole-cell FQ current decay.

The fitting algorithm determines a set of rate constants that minimize the difference between the calculated probability of open-state occupancy and the measured open-state probability. Whole-cell currents at $-20 \mathrm{mV}$ were scaled into open-state probability curves using peak open-state probabilities measured in cell-attached patches (31). A numerical integration method for solving stiff sets of differential equations was utilized to calculate open state probabilities (LSODA) $(32,33)$ from a best-estimate set of initial rate constants. Data at multiple drug concentrations were fitted simultaneously to gating models that differed only in the rate constants altered by the concentration of drug (see Fig. 9). The inverse sum of the rate constants exiting the open state was limited by the measured mean open time at $-20 \mathrm{mV}$. A modified Simplex algorithm (34) was used to iteratively modify the rate constants while minimizing the residual sum of squares. The fitting procedure restarts from multiple sets of initial rate constants before ultimately converging at a final solution.

\section{Results}

Lidocaine effects on whole-cell currents from inactivation-defective Na channels. Whole-cell currents recorded from wildtype Na channels expressed in Xenopus oocytes decay rapidly, whereas FQ channels exhibit slower decay and a sizable persistent plateau current at the end of a $100 \mathrm{~ms}$ depolarizing pulse to $-20 \mathrm{mV}$ (Fig. $1 A$ ), as shown previously (35) for the analogous human cardiac (hH1) Na channel mutant. The half-time of current decay (the interval from the current peak to $50 \%$ reduction of the current amplitude) was $5.9 \pm 2.3 \mathrm{~ms}$ in wild-type channels $(n=40)$ vs. $45.9 \pm 23.3 \mathrm{~ms}$ in FQ channels $(n=25$, $P<0.0001)$. Lidocaine added to the bath solution produced dose-dependent blockage of both wild-type (left) and FQ (right) Na currents during step depolarizations to $-20 \mathrm{mV}$ (Fig. $1 \mathrm{~A}$ ). For FQ channels, the plateau current was blocked much more potently than was the peak current. Indeed, at a concentration of $300 \mu \mathrm{M}$, lidocaine nearly restored wild-type inactivation to FQ whole-cell current. The dose-response relationships for both channels are shown in Fig. $1 \mathrm{~B}$. The mean current scaled to the drug-free value is plotted for the peak inward current (wild-type and FQ) and the plateau current (FQ only, quantified $100 \mathrm{~ms}$ after stepping to $-20 \mathrm{mV}$ ). The peak current dose-response curves for FQ (solid triangles) and wildtype (solid squares) were similar. However, the FQ plateau current (open triangles) was nearly an order of magnitude more sensitive to lidocaine $\left(\mathrm{IC}_{50}=74 \mu \mathrm{M}\right.$ vs. $513 \mu \mathrm{M}$ for FQ peak, $P<0.005)$.

Wild-type Na channels exhibit modal gating, consisting of a predominant fast mode and a lower probability slow mode. The prevalence of slow mode gating greatly influences the rate of decay of wild-type whole-cell or ensemble average currents, and saturating coexpression of the $\beta_{1}$ subunit in Xenopus oocytes nearly eliminates this slow gating mode (36-39) and ac-

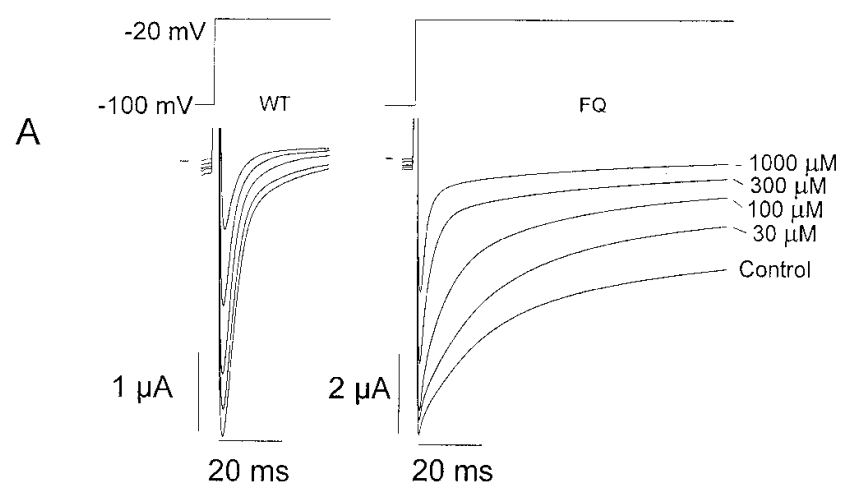

B

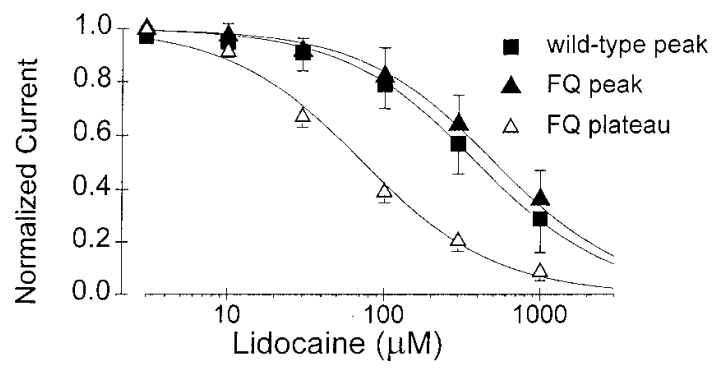

Figure 1. Lidocaine effects on whole-cell currents recorded from wild-type and FQ mutant $\mu 1 \mathrm{Na}$ channels expressed in Xenopus oocytes. (A) Currents recorded from an oocyte expressing wild-type $\alpha$ subunits (left) and another oocyte expressing FQ $\alpha$ subunits (right). The voltage clamp protocol is shown above the current traces. When added to the bath solution, lidocaine produced a dose-dependent current reduction. The drug concentrations used in the wild-type and FQ experiments were the same (shown beside the FQ currents: 0, 30, 100, $300,1000 \mu \mathrm{M})$. (B) The dose-response relationships for wild-type peak current (filled squares; $n=8$ oocytes) and FQ peak (filled triangles) and plateau (open triangles) current at $100 \mathrm{~ms}$ ( $n=5$ oocytes) are plotted as mean \pm SD. The peak and plateau dose-response data were leak-subtracted and normalized to the control value measured in the same oocyte, and were then fitted to a logistic function $1 /(1+$ $\left.\mathrm{IC}_{50}\right)$. The solid lines are least-squares fits to the mean data.

celerates the decay of whole-cell current $(23,24)$. We have recently shown that FQ channels also fluctuate between two principal modes (bursting and nonbursting), that this mode shifting influences the decay of FQ ensemble average current, and that $\beta_{1}$ coexpression reduces the probability of bursting sweeps in cell-attached patches from $15 \%$ to $<1 \%$ (31). Therefore, it was important to determine whether the lidocaineinduced acceleration of FQ whole-cell current decay was the result of a selective interaction of drug with channels occupying the bursting mode. To exclude this possibility, the experiments of Fig. 1 were repeated with $\beta_{1}$ subunit coexpression. Fig. 2, $A$ and $B$ show the dose-dependent effect of lidocaine on whole cell currents from wild-type and FQ channels coexpressed with the $\beta_{1}$ subunit $\left(\mathrm{FQ}+\beta_{1}\right)$. The dose-response curves are shown for peak and 100 ms plateau currents in Fig. $2 D$. As before, lidocaine accelerated the decay of $\mathrm{FQ}+\beta_{1}$ current; the 100-ms plateau current was blocked with an $\mathrm{IC}_{50}$ of $280 \mu \mathrm{M}$, while the peak current was blocked with a higher $\mathrm{IC}_{50}$ of $1570 \mu \mathrm{M}(P=0.008)$. Block of the FQ $+\beta_{1}$ peak current was not significantly different from that of wild-type $+\beta_{1}$ $\left(\mathrm{IC}_{50}=1230 ; P=0.08\right.$ versus $\left.\mathrm{FQ}\right)$. The dose response curves for lidocaine block of peak and plateau FQ and wild-type cur- 


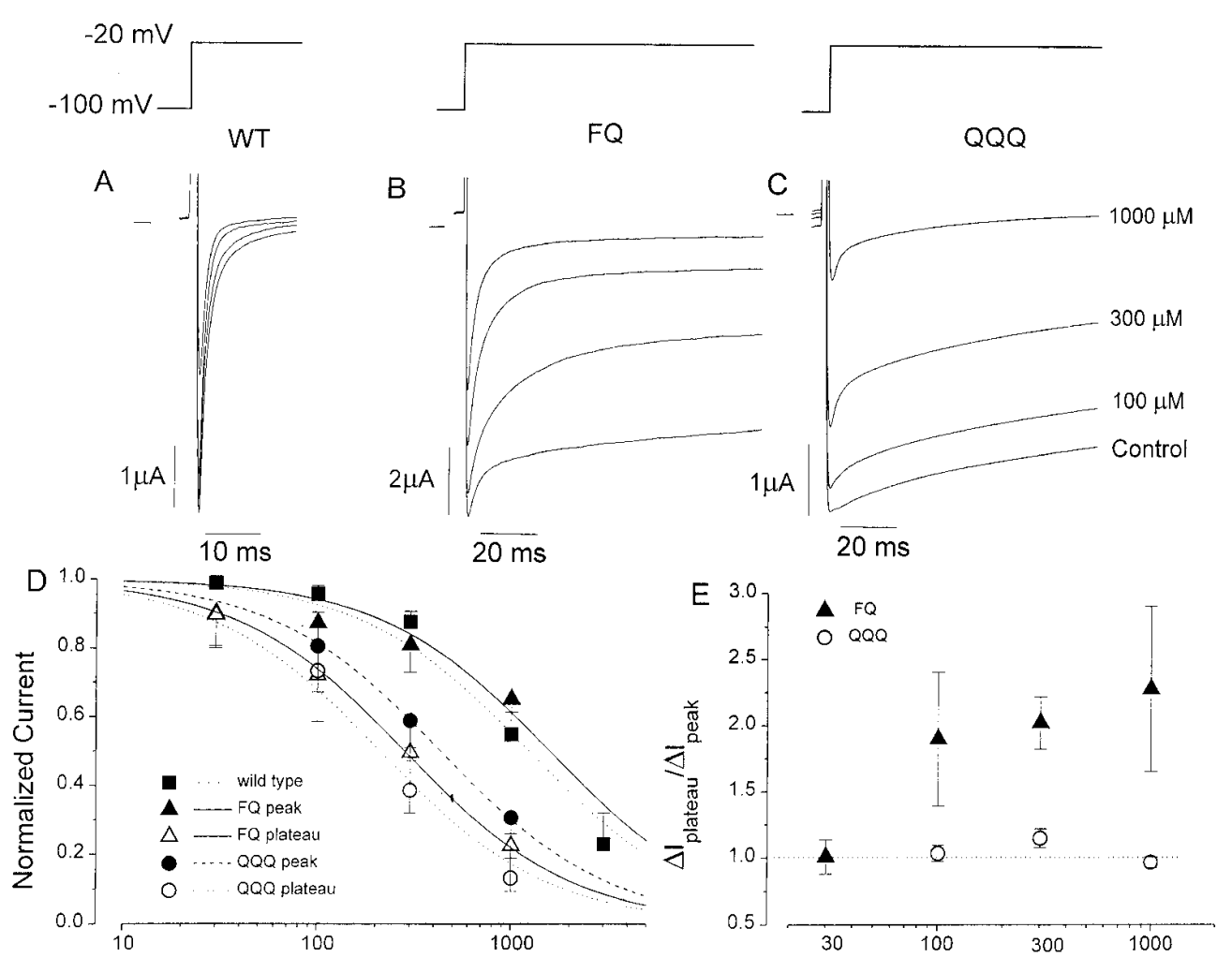

Figure 2. Comparison of lidocaine effects on wild-type, FQ and QQQ channels with the $\beta_{1}$ subunit coexpressed. Wholecell currents were recorded from oocytes expressing wild-type $(A), \mathrm{FQ}(B)$, and QQQ channels $(C)$. The currents were recorded in bath lidocaine concentrations of $0,100,300$, and $1000 \mu \mathrm{M}$ (shown beside the QQQ currents). (D) Lidocaine doseresponse curves for peak and plateau current reduction in wild-type $(n=15)$, FQ $(n=6)$, and QQQ $(n=7)$ oocytes all with $\beta_{1}$ subunit coexpressed. The data were normalized to the control current in the same oocyte, and were plotted (mean $\pm \mathrm{SD})$. The mean values were fitted to the logistic function and are plotted as in Fig. 1. These fitted $\mathrm{IC}_{50}$ values were $(\mu \mathrm{M})$ : wild-type $=1230 ; \mathrm{FQ}$ peak $=1572 ;$ FQ plateau $=280$; QQQ peak $=431$; QQQ plateau $=210 .(E)$ The ratio of lidocaine-blocked plateau current $\left(\Delta \mathrm{I}_{\text {plateau }}\right)$ to lidocaine-blocked peak current $\left(\Delta \mathrm{I}_{\text {peak }}\right)$ is plotted as a function of lidocaine concentration (mean $\left.\pm \mathrm{SE}\right)$ for the same oocytes as in $D$. At all concentrations, lidocaine block of QQQ current at $100 \mathrm{~ms}$ was similar to block of peak current. In contrast, the FQ block ratios were consistently $>1$ at doses $\geq 100 \mu \mathrm{M}$ due to greater reduction of plateau current than peak current.

rents are noticeably shifted rightward by $\beta_{1}$ coexpression (compare to Fig. 1). The magnitude of this shift is nearly identical to that reported for lidocaine block of human heart $(\mathrm{hH} 1)$ Na currents (40), where block of peak current was shifted by $\beta_{1}$ coexpression from 500 to $1400 \mu \mathrm{M}$. An effect of $\beta_{1}$ on the lidocaine receptor has been proposed (40) as the mechanism of this shift in lidocaine dose-response.

The prominent acceleration of FQ whole-cell current decay by lidocaine even with $\beta_{1}$ subunit coexpression suggests that lidocaine must act on channels gating in the predominant, nonbursting (31) mode. However, the mechanism by which lidocaine interacts with the FQ channel still remains ill-defined. The acceleration of FQ current decay may result from blockade of the open pore in a manner similar to tetraethylammonium block of delayed rectifier potassium channels (41). Alternatively, lidocaine may accelerate FQ current decay through an interaction with $\mathrm{Na}$ channel inactivation. To discriminate between these mechanisms, we used three complementary approaches. First, we compared the dose-dependent effect of lidocaine on channels with inactivation partly disabled (FQ) to channels with inactivation more fully disabled (QQQ). We reasoned that if open-channel block is the primary mechanism for acceleration of FQ current decay, this effect should not be attenuated by a more severe lesion of the inactivation gating apparatus. Second, to evaluate the open-channel kinetics of local anesthetic block more specifically, we measured singlechannel FQ currents and examined the kinetic properties of open-channel block. Finally, quantitative modeling was utilized to examine whether inactivated-state or open-state modulated receptor models could simulate these data.
Comparison of lidocaine effects on $Q Q Q$ channels to $F Q$ channels. Fig. 2, $B$ and $C$ compare the effect of lidocaine on FQ and QQQ currents. Inspection of the early decay of current through FQ and QQQ channels in the absence of drug confirms the idea that inactivation is nearly completely eliminated by the triple (QQQ) mutation (13). In contrast to FQ, QQQ currents have less drug-induced decay during the depolarizing clamp step. Examination of the peak and plateau doseresponse relationships (Fig. $2 \mathrm{D}$ ) indicates that the $\mathrm{IC}_{50}$ for lidocaine block of QQQ peak current $(430 \mu \mathrm{M})$ was similar to that of QQQ plateau $(210 \mu \mathrm{M})$ current, and these $\mathrm{IC}_{50}$ values did not differ significantly from the $\mathrm{IC}_{50}$ for $\mathrm{FQ}$ plateau current $(P=0.075)$. The similar sensitivity of peak and plateau QQQ currents to lidocaine differs markedly from the drug effects on FQ, and partly explains the attenuated drug-induced decay of QQQ current compared to FQ. We quantified the druginduced decay for both mutants by plotting the ratio of blocked plateau current to blocked peak current over a range of lidocaine concentrations (Fig. 2 E). The line of unity (dotted line) indicates the predicted result for no drug-induced current decay. These results indicate that the time-dependent effect of lidocaine on FQ currents during a depolarizing pulse differs qualitatively from that for QQQ currents. This difference argues that the effect of lidocaine on FQ whole-cell current decay is linked to the inactivation mechanism, rather than being dependent on slow blockade of the open pore.

Lidocaine restores use dependence to $F Q$ channels. As previously noted, $300 \mu \mathrm{M}$ lidocaine restored the rapid decay of whole-cell current to FQ channels (Fig. $1 B$ ). If this occurred through stabilization of the inactivation process, lidocaine 
should also delay recovery from inactivation in FQ channels, and thereby induce cumulative inactivation during a rapid train of depolarizing pulses. Previous studies of human cardiac $\mathrm{Na}$ channels with inactivation severely disabled by the QQQ mutation showed that exposure to lidocaine did not induce use dependence during a $10-\mathrm{Hz}$ train (14). However, the greater lidocaine sensitivity of FQ plateau current relative to QQQ suggested that lidocaine might enhance use dependence in FQ channels.

Fig. $3 \mathrm{~A}$ shows pairs of currents recorded from oocytes coexpressing FQ and $\beta_{1}$ subunits. Currents were measured during consecutive $50-\mathrm{ms}$ depolarizing pulses to $-20 \mathrm{mV}$ from $-100 \mathrm{mV}$ at a frequency of $10 \mathrm{~Hz}$. Currents are shown before drug exposure (Fig. 3 A, left) and during superfusion with lidocaine (right). In the absence of lidocaine, FQ channels exhibit very little use-dependent reduction in peak current. However, with lidocaine present, the peak current during the second pulse was significantly reduced relative to the first pulse. Like wild-type channels exposed to lidocaine, FQ channels exhibited delayed recovery from inactivation. Recovery from inactivation is analyzed in detail in Fig. 3 B. After a 50-ms inactivating prepulse (P1), recovery from inactivation after increasing repriming intervals was assessed using a test pulse (P2). As shown previously (42), FQ channels recover from inactivation very rapidly $(<10 \mathrm{~ms}$, open circles) in the absence of drug. In the presence of lidocaine (filled squares), complete recovery from inactivation was delayed for $\sim 500 \mathrm{~ms}$. This delay in recovery was greater than that seen in drug-free solutions even for wild-type $\left(\alpha+\beta_{1}\right)$ channels with intact fast inac- tivation (43). Thus, lidocaine not only accelerates FQ inactivation (Fig. $1 A$ ), but also stabilizes inactivation, causing a delay in recovery from inactivation and enhanced use dependence.

Lidocaine effects on single FQ channels. Single-channel recordings are the most sensitive and direct means to examine open channel block. In the absence of drug, cell-attached single-channel recordings (Fig. $4 A$ ) reveal that, relative to wild-type channels, FQ channels open much more frequently during each depolarization. The ensemble average currents assembled from single-channel records (Fig. $4 B$ ) reconstitute the slow decay seen in whole-cell FQ recordings (Fig. $1 A$ ). Application of $300 \mu \mathrm{M}$ lidocaine to FQ channels accelerated the decay of ensemble average current (Fig. $4 \mathrm{~B}$ ), confirming the results from whole-cell recordings. Inspection of singlechannel activity during individual FQ records (Fig. $4 A$ ) reveals that reopenings were less frequent in patches exposed to lidocaine. The mean number of openings per FQ channel during 100 consecutive depolarizing pulses (excluding blank sweeps) was reduced by lidocaine from $16.3 \pm 0.8$ to $8.7 \pm 0.5$ $(P<0.0001)$.

Mean open times for FQ single channels were prolonged relative to wild-type $(31,35,42)$, but were still well-described by a single exponential $(31,42)$. Drug association with the open channel at a rate comparable to intrinsic wild-type inactivation should be apparent as a reduction in the mean open time. Fig. $5 A$ shows that FQ mean open time was minimally altered by $300 \mu \mathrm{M}$ lidocaine over a wide range of membrane potentials $(-60$ to $+10 \mathrm{mV})$. At $-10 \mathrm{mV}$, the mean open time was $2.47 \pm 0.1 \mathrm{~ms}$ in control solutions $(n=4)$, and $2.13 \pm 0.4 \mathrm{~ms}$

A

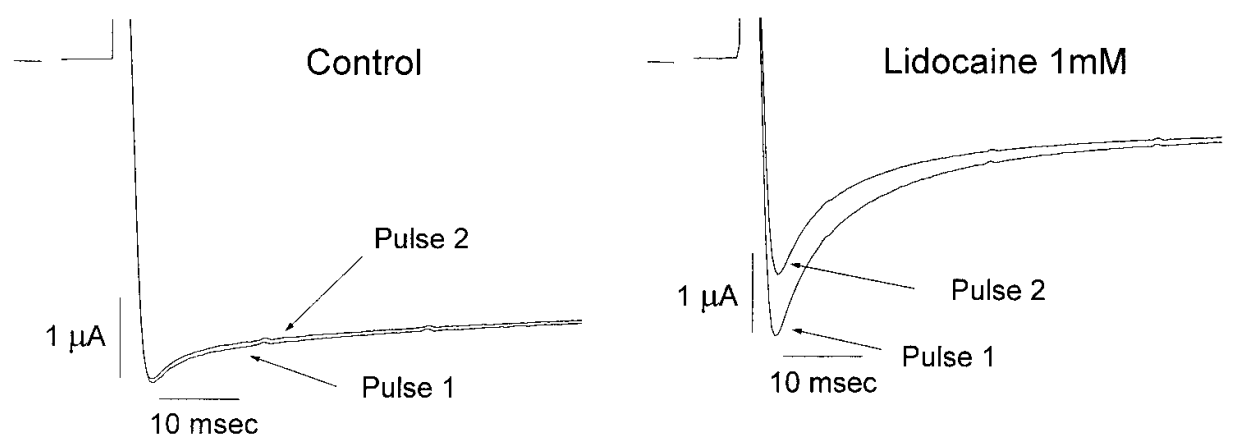

Figure 3. Lidocaine delays recovery from $\mathrm{FQ}+\beta_{1}$ inactivation. $(A) \mathrm{FQ}+\beta_{1}$ currents measured during two consecutive 50-ms depolarizing pulses separated by a $100 \mathrm{~ms}$ recovery interval at $-100 \mathrm{mV}$. In the absence of drug (left), channels recover fully from inactivation during the repriming interval, so that the measured currents are nearly identical. With lidocaine $(1 \mathrm{mM})$ in the bath solution, the peak current measured during the second pulse is significantly reduced. $(B)$ Recovery from inactivation was assessed in greater detail using the pairedpulse voltage-clamp protocol shown. After depolarizing oocytes for $50 \mathrm{~ms}(P 1)$, fractional recovery during a subsequent test pulse (P2) was assessed after an intervening recovery interval. Drug-free channels recovered rapidly in less than 50 ms ( $n=4$ oocytes), but lidocaine delayed recovery of FQ channels for $\geq 500 \mathrm{~ms}(n=6$ oocytes). 


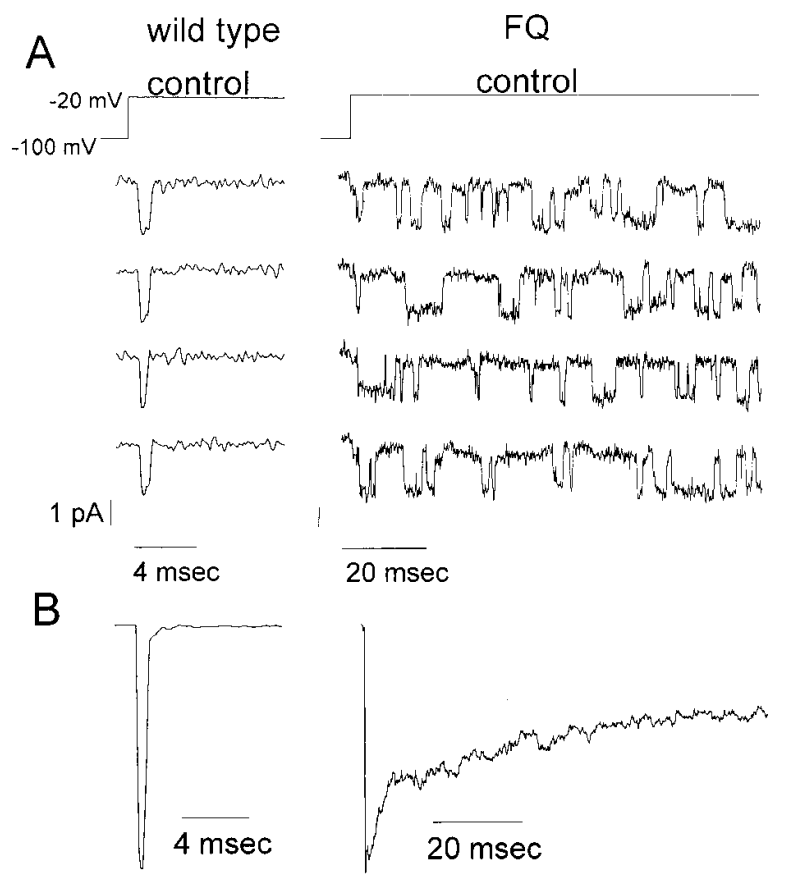

in lidocaine $(n=4)$. To exclude the possibility that $\beta_{1}$ subunit coexpression modifies the effect of lidocaine on FQ open time through a mode shift, we performed additional cell-attached patch experiments with FQ $\alpha$ subunits coexpressed with $\beta_{1}$. At $-10 \mathrm{mV}$, mean open times were minimally reduced from $2.30 \pm 0.1 \mathrm{~ms}$ in control solutions $(n=3)$ to $2.22 \pm 0.5 \mathrm{~ms}$ in 300 $\mu \mathrm{M}$ lidocaine $(n=3)$. These results suggest that the lidocaineinduced decay of FQ whole-cell current does not result from either an open-channel block mechanism (for review see reference 41) or a shift in gating modes.

A reduction in unitary current amplitude was apparent in the single-channel records from patches exposed to lidocaine (Fig. $4 A$ ), consistent with very rapid open-channel block with kinetics faster than the bandwidth of our recording apparatus (44). Unitary FQ current amplitudes recorded in the absence and presence of lidocaine are plotted as a function of membrane potential in Fig. 5 B (left). While the absolute reduction in unitary current amplitude by lidocaine is similar at each voltage, the fractional reduction in unitary current increased with depolarization in the manner predicted for intrapore binding of a cation moving in the membrane electric field from the cytoplasmic face (45). A logarithmic transformation of the blocked and unblocked current amplitudes (Fig. 5 B, right) allowed estimation of an electrical distance to the binding site ( $\delta$ ) of 0.68 from the cytoplasmic face, and an affinity at $0 \mathrm{mV}$ $\left(\mathrm{K}_{\mathrm{d} 0}\right)$ of $877 \mu \mathrm{M}$. This low affinity for the open pore differs from estimates of lidocaine affinity for the inactivated state $\left(K_{\mathrm{d}} \sim 1-12 \mu \mathrm{M}\right)(4,9,46)$, but is consistent with other analyses of fast voltage-dependent block of the open channel (47). Rapid intrapore block reduces the unitary current amplitude and scales the ensemble average current to some degree, but would not accelerate the macroscopic current decay.

Rapid block of $F Q$ channels by $Q X-314$. At neutral $\mathrm{pH}$, lidocaine exists as a mixture of charged and uncharged species. The charged species is responsible for the rapid low-affinity intrapore block (Fig. $4 A$ ), and does not explain the lidocaine-

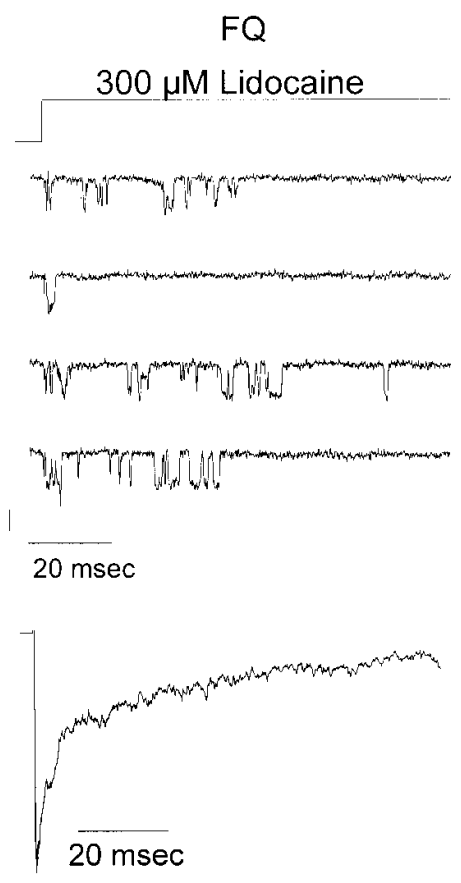

Figure 4. Representative singlechannel currents recorded from cell-attached patches in Xenopus oocytes. $(A)$ Wild-type (left), FQ (middle), and FQ with $300 \mu \mathrm{M}$ lidocaine in the patch electrode (right). Currents were elicited by membrane depolarization from $-120 \mathrm{mV}$ to -20 $\mathrm{mV}$ at a frequency of $0.67 \mathrm{~Hz}$. The wild-type patch contained two channels, the FQ patch contained one channel, and the FQlidocaine patch contained two channels. ( $B)$ Normalized ensemble average currents were derived from the patches shown in $A$. The ensemble currents were assembled from (left to right) 516, 1000, and 518 idealized records, respectively. induced FQ current decay. Must this time-dependent effect of lidocaine then result exclusively from the uncharged species? To assess this, the permanently charged lidocaine derivative QX-314 was studied in inside-out patches containing FQ channels. We reasoned that if the "hydrophobic pathway" (2) was solely responsible for the time-dependent effect of lidocaine on FQ current, QX-314 should exhibit rapid, low-affinity poreblocking characteristics, but should not accelerate ensemble average current decay or significantly reduce the probability of channel opening. Fig. $6 \mathrm{~A}$ shows selected sweeps at step potentials from $-60 \mathrm{mV}$ to $0 \mathrm{mV}$ before (left) and during (right) exposure to $2 \mathrm{mM}$ QX-314. The single-channel records suggest that QX-314 blocks the open pore in a manner qualitatively similar to lidocaine. Unitary current amplitudes from a patch sequentially exposed to control solutions and two concentrations of QX-314 are plotted as a function of transmembrane potential (Fig. $6 \mathrm{~B}$ ) and analyzed as for lidocaine in Fig. $5 \mathrm{~B}$. The affinity of the channel for QX-314 at $0 \mathrm{mV}$ was low $\left(K_{\mathrm{d} 0}=\right.$ 2.0-2.4 $\mathrm{mM}$ ), and the estimated electrical distance to the binding site was similar to that of lidocaine $(\delta=0.51-0.62$, Fig. $6 B)$. QX-314 block of excised FQ channels expressed in HEK-293 cells was similar to that measured in oocytes (data not shown, $\left.\delta=0.51, K_{\mathrm{d} 0}=2.0 \mathrm{mM}\right)$.

We next examined whether QX-314 blocks the open channel with slower kinetics, on the order of the wild-type inactivation rate. Fig. 7, $A$ and $B$ show open time histograms constructed from oocyte data obtained before and during exposure to QX314. Similar to the results with lidocaine (Fig. $5 \mathrm{~A}$ ), there was no significant effect of $0.5 \mathrm{mM}$ QX-314 on the mean open time at $-40 \mathrm{mV}$ (control $=1.4 \mathrm{~ms}, \mathrm{QX}-314=1.3 \mathrm{~ms}$ ) or at $-60 \mathrm{mV}$ (control $=0.8 \mathrm{~ms}, \mathrm{QX}-314=0.9 \mathrm{~ms}$; data not shown). However, while $0.5 \mathrm{mM}$ QX-314 produced minimal open-channel block effects (see also Fig. 6 B left, filled squares), the effect on the probability of channel opening was substantial. Fig. $7 C$ is a diary from an experiment showing the probability of channel opening during repeated depolarizing steps to $-40 \mathrm{mV}$. At $0.5 \mathrm{mM}$, 


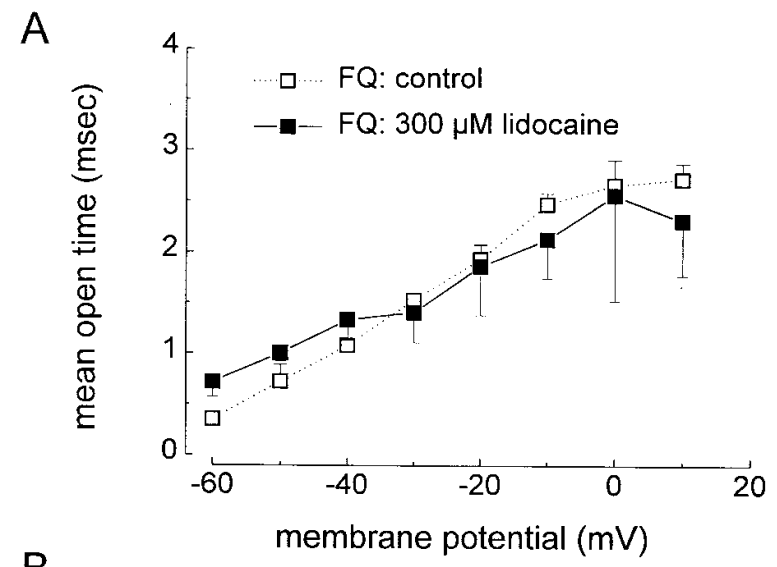

$B$
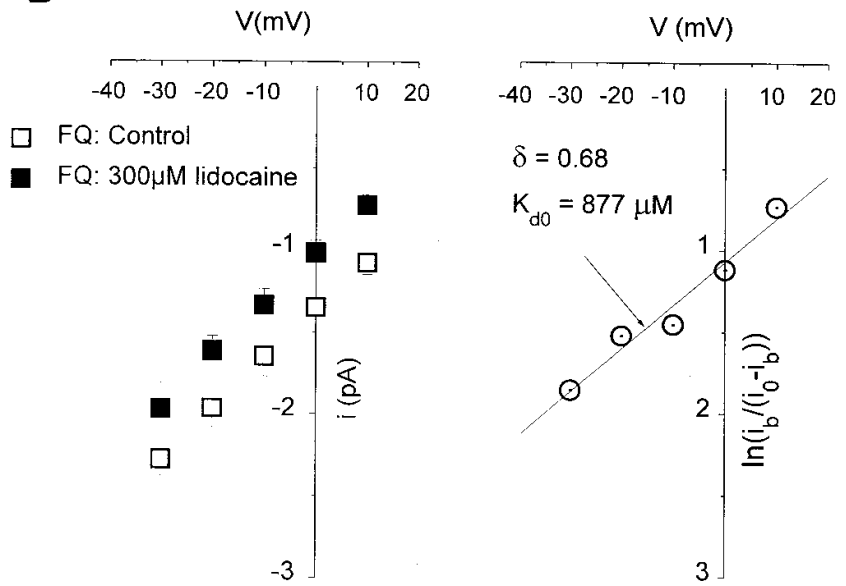

Figure 5. Analysis of open channel block by lidocaine. $(A)$ Mean open times (mean $\pm \mathrm{SD}$ ) determined from single-exponential fits to open-time histograms composed from unitary currents measured during repeated step depolarizations to $-20 \mathrm{mV}$. Data are shown for FQ (open squares, 3 patches) and FQ with lidocaine (filled squares, 4 patches). ( $B$, left) Unitary FQ currents (mean \pm SD) from the same patches as in $A$ are plotted as a function of membrane potential in the absence (open squares) and presence (filled squares) of lidocaine. Dotted lines are linear fits to the mean data. (Right) The blocked $\left(\mathrm{i}_{\mathrm{b}}\right)$ and unblocked $\left(\mathrm{i}_{0}\right)$ mean current amplitudes were replotted as $\mathrm{y}=\ln$ $\left[\mathrm{i}_{\mathrm{b}} /\left(\mathrm{i}_{0}-\mathrm{i}_{\mathrm{b}}\right)\right]$. The slope and $\mathrm{y}$ intercept of the best linear fit to the transformed data were then used to estimate the electrical distance to the binding site from the cytoplasmic face $(\delta)$ and the affinity of the channel for lidocaine at $0 \mathrm{mV}\left(\mathrm{K}_{\mathrm{d} 0}\right)(45)$.

QX-314 significantly reduced the channel activity, and increasing to $2 \mathrm{mM}$ QX-314 actually made channel openings rare. The drug effect was partially reversible (Fig. $7 C$ ). Fig. $7 D$ shows the effect of $0.5 \mathrm{mM}$ QX-314 on ensemble average current at -40 $\mathrm{mV}$. Similar to lidocaine (Fig. $1 A$ ), QX-314 hastened the decay and reduced the plateau of the ensemble average current. Although QX-314, like lidocaine, exhibits rapid pore block, the effect is small at doses which substantially reduce the probability of channel opening and accelerate the decay of ensemble average current. These results suggest that the charged lidocaine species contributes to both low affinity open channel block as well as a higher affinity inactivation-enhancing mechanism.

A model for lidocaine block. Modulated receptor models for local anesthetic block that are based on a simple 3-state $\mathrm{Na}$ channel gating model (Eq. 1) only consider drug binding to channels that are fully activated (open) or inactivated; hence,
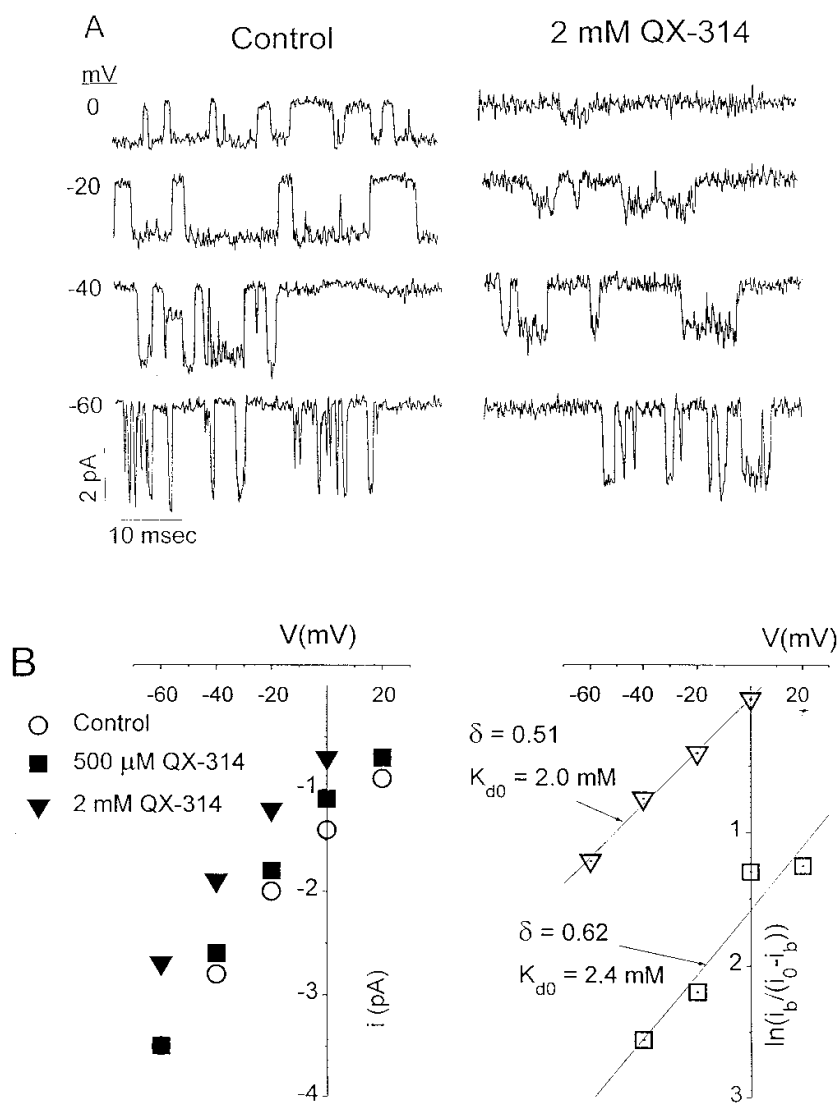

Figure 6. Open-state block of FQ channels. ( $A$ ) Single FQ currents recorded from inside-out excised patches at four voltages are shown in the absence (left) and presence (right) of $2 \mathrm{mM} \mathrm{QX-314.} \mathrm{The} \mathrm{effect}$ of $2 \mathrm{mM} \mathrm{QX}-314$ is a voltage-dependent reduction in unitary current amplitude. The sample rate was $10 \mathrm{kHz}$, and data were filtered at 2 $\mathrm{kHz}$. The patch contained 4 channels. $(B$, left) Unitary current amplitudes measured from the same patch before exposure to drug (open circles), and during exposure to $0.5 \mathrm{mM}$ (filled squares) and $2.0 \mathrm{mM}$ QX-314 (filled triangles). The effects of QX-314 on unitary current amplitude were fully reversible. (Right) Fractional electrical distance to the site of block and the channel affinity for QX-314 were calculated as in Fig. 5 B. Calculations based on current amplitudes measured in $0.5 \mathrm{mM}$ (open squares) and $2.0 \mathrm{mM} \mathrm{QX}-314$ (open triangles) yielded similar results.

any rapidly developing block component is explained by high affinity for the open-channel (4).

Drug-free: $\quad$ Rested $\underset{n}{ } \underset{n}{\operatorname{Open}} \underset{n}{\stackrel{\text { Inactivated }}{n}}$

Drug-associated: $\quad *$ Rested $\rightleftarrows$ Open $\rightleftarrows$ Inactivated

In their original work, Hodgkin and Huxley (48) deduced that distinct processes control $\mathrm{Na}$ channel opening and inactivation. Later single-channel measurements confirmed that $\mathrm{Na}$ channels need not open to inactivate $(49,50)$; while some channels may inactivate after opening, a significant fraction inactivate directly from closed states during the channel activation process. However, $\mathrm{Na}$ channel gating current measurements (51) and structure-function studies of paramyotonia congenita mutations (52) have confirmed that inactivation is at least partially coupled to activation. Contemporary models of $\mathrm{Na}$ channel gating thus define the coupling between activation 

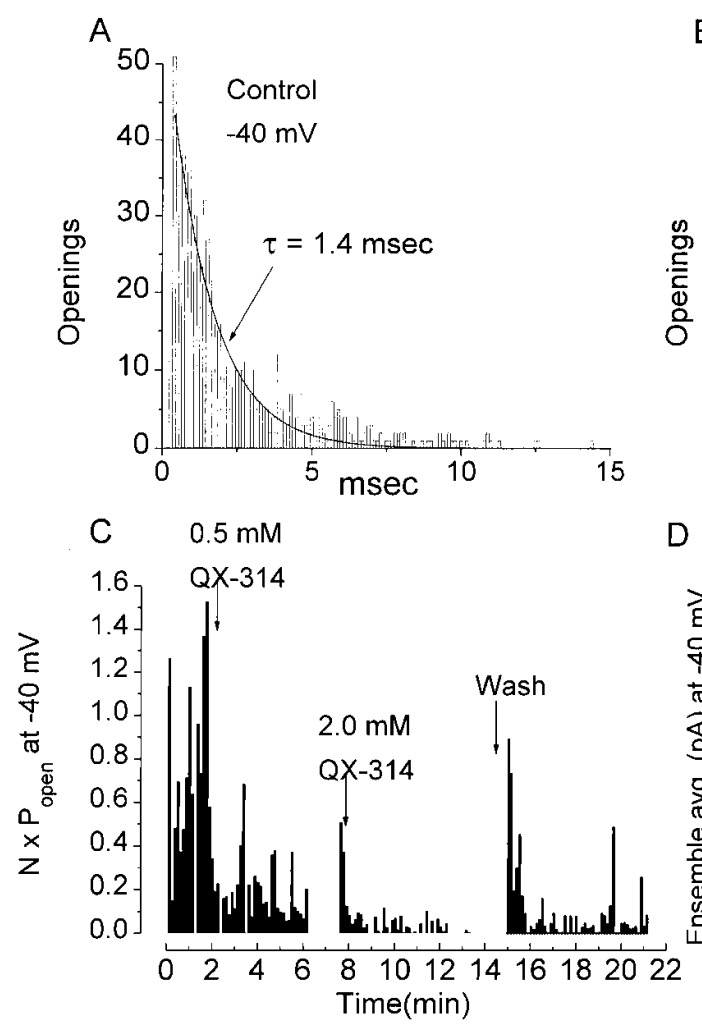
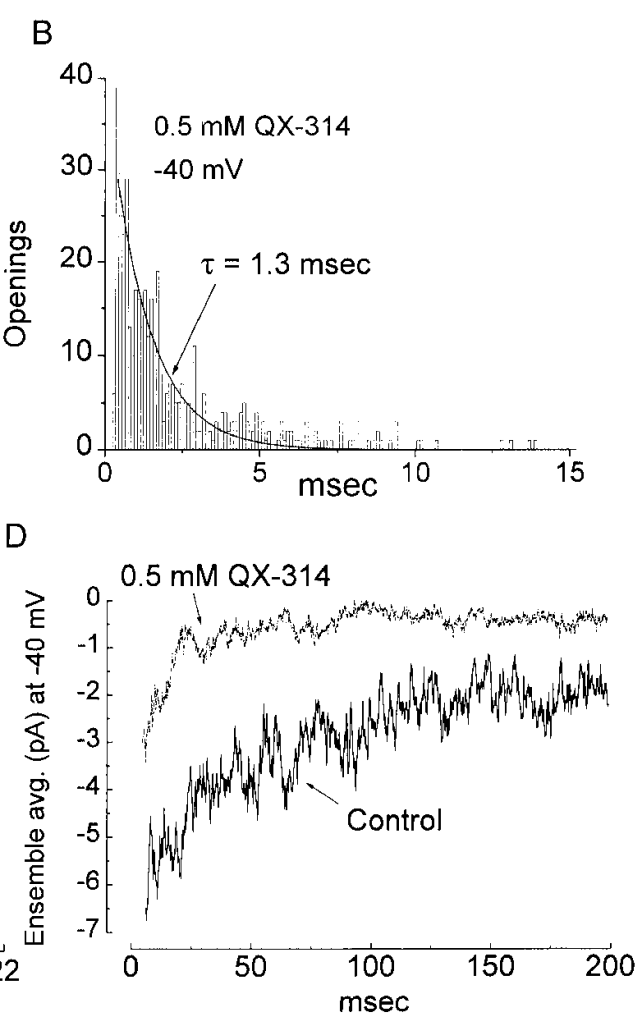

Figure 7. The effect of QX-314 on mean open time and probability of channel opening. ( $A$ and $B$ ) QX-314 does not significantly change channel open times. Open time histograms are shown for FQ in the absence and presence of $0.5 \mathrm{mM} \mathrm{QX}-314$ at $-40 \mathrm{mV}$. The bin size $=0.1$ $\mathrm{ms}$, and the superimposed solid lines are least-squares single exponential fits. $(C)$ Activity diary of an oocyte patch during a control period, after exposure to 0.5 mM QX-314, then $2.0 \mathrm{mM}$ QX314 , followed by a washout period. The patch was depolarized from $-120 \mathrm{mV}$ to $-40 \mathrm{mV}$ for $280 \mathrm{~ms}$ at a 1.5 -s repetition interval. The patch activity $(\mathrm{N} \times$ $\mathrm{P}_{\text {open }}$ ) reflects the product of the probability of a single-channel opening and the number of channels in the patch; in this patch, $n=4$ channels. QX-314 reduced the likelihood of channel opening in a concentrationdependent manner and was partially reversible. $(D)$ Ensemble average of the nonidealized sin-

gle-channel current for the patch described in C. QX-314 at a $0.5 \mathrm{mM}$ concentration hastens the ensemble average current decay. The control ensemble average contains 16 sweeps; the drug-exposed ensemble average is from the same oocyte and contains 30 sweeps.

and fast inactivation explicitly $(16,53,54)$, allowing pharmacologic or gating interactions throughout the activation process.

Mutagenesis experiments have suggested that fast inactivation of Na channels results from closure of the cytoplasmic III-IV linker over the inner mouth of the pore $(13,55-59)$. Consistent with this interpretation, a recent analysis treated fast inactivation as a particle-binding process coupled in parallel to activation (Eq. 2) (16). The activation process was modeled as movement of four independent, identical voltage sensors, followed by an additional opening step. The inactivation rate constants were multiplied by a scaling factor $(a)$ which increased the affinity of the channel for the inactivation particle as it progressed through the activation sequence, but at the same time allowed a significant fraction of channels to inactivate early from closed states. This type of coupling between activation and inactivation closely resembles a gating model for calcium channel activation and dihydropyridine agonist effects (60), where activation and channel opening were coupled using a Monod-Wyman-Changeux paradigm for cooperativity in allosteric proteins (61).

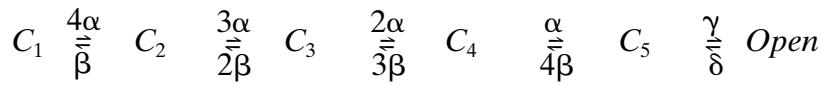

$$
\begin{aligned}
& k_{1} 1 \imath k_{2} \quad k_{1} / a \imath \imath k_{2} a \quad k_{1} / a \imath \imath k_{2} a^{2} \quad k_{1} / a^{3} \imath \iota k_{2} a^{3} \quad k_{1} / a^{4} 1 \imath k_{2} a^{4} \quad k_{3} \imath \iota k^{4}
\end{aligned}
$$

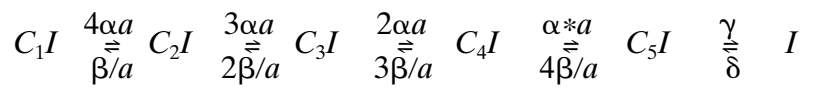

$$
\begin{aligned}
& a=\left[\left(k_{1} / k_{2}\right) /\left(k_{3} / k_{4}\right)\right]^{1 / 8}
\end{aligned}
$$

Using this model we simulated wild-type and FQ macroscopic currents in the absence of drug (Fig. 8). The activation/ deactivation ( $\alpha, \beta, \gamma, \delta$ : horizontal) rate constants (Fig. 8, bottom) for wild-type and FQ were identical, while the inactivation rate constants $(\mathrm{k} 1, \mathrm{k} 2, \mathrm{k} 3, \mathrm{k} 4$ : vertical) differed for wild-type and FQ to reflect the dual effect of the mutation on the inactivation gate association $\left(\mathrm{k}_{2}\right.$ and $\left.\mathrm{k}_{4}\right)$ and dissociation $\left(\mathrm{k}_{1}\right.$ and $\left.\mathrm{k}_{3}\right)$ rates $(31,35,62)$. For $F Q, k_{1}$ and $k_{3}$ are increased, while $k_{4}$ is decreased relative to wild-type. Based on these rate constants, the predicted drug-free mean open times at $-20 \mathrm{mV}$ were 0.44 ms for wild-type and $1.7 \mathrm{~ms}$ for FQ, consistent with measured values in cell-attached patches (31).

To examine whether local anesthetics alter $\mathrm{Na}$ channel gating by influencing coupling between gating processes, we first tested whether modulated receptor schemes that ignore such an effect could reproduce the effects of lidocaine on FQ currents (Fig. 9). Model A examines the possibility that lidocaine binds to the activating channel as an independent blocking particle, with increasing affinity as the channel progresses toward opening. This model is the analogue of the open-state block model employing the classical Hodgkin-Huxley gating paradigm. Kuo and Bean (16) simulated recovery from diphenylhydantoin block in this manner, and imparted slower unbinding kinetics to drug than to the native inactivation particle. Although this framework was adequate for reproducing FQ currents at particular lidocaine concentrations, Fig. 9 (bottom, left) shows that it was not possible to simulate drug effects over a broad range of drug concentrations with the same kinetic model. Rate constants that reasonably simulate the currents measured in $300 \mu \mathrm{M}$ lidocaine cause an implausible overshoot at $30 \mu \mathrm{M}$ 


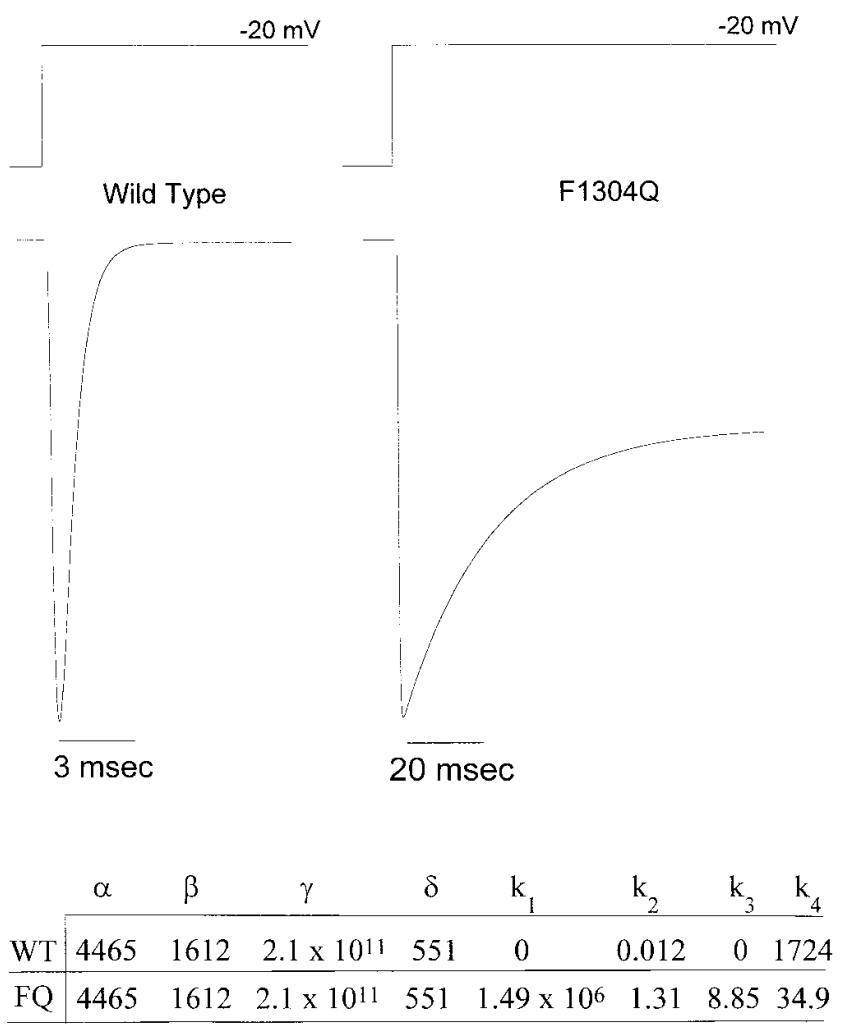

Figure 8. A contemporary gating model for inactivation-disabled $\mathrm{Na}$ channels. Simulated macroscopic currents are shown for wild-type (left) and FQ (right) Na channels using a model described by Kuo and Bean (16) (Eq. 2). The model was iteratively adjusted to find an optimal set of rate constants using a stiff differential equation solver and a Simplex parameter search algorithm as described previously (30). The parameter search was constrained by the peak open-channel probabilities (data not shown) and mean open times (31) to produce simulations consistent with kinetic parameters determined from single-channel data. These constraints provided simulated currents with peak open probabilities of 0.21 and 0.54 for wild-type and FQ channels, respectively (the simulations as shown are normalized to match the two peaks). The resultant wild-type and FQ model rate constants are shown at the bottom; note that only the inactivation rates (vertical rate constants, Eq. 2) were allowed to differ for the wild-type and FQ models.

(Fig. $1 A$ ). Further, in order to simulate the effect of lidocaine on macroscopic FQ at concentrations $\geq 300 \mu \mathrm{M}$, drug on-rates were so high that they reduced the predicted mean open time by $42 \%$, inconsistent with our single-channel results (Fig. $5 \mathrm{~A}$ ).

Model B (Fig. 9) examines high-affinity lidocaine binding to the inactivated channel. The analogous modulated-receptor model based on a traditional Hodgkin-Huxley gating scheme is shown for comparison. This model has historical significance as a successful paradigm for explaining use-dependence and delayed recovery from inactivation for lidocaine-exposed $\mathrm{Na}$ channels. In the limit (infinitely high lidocaine concentration), such binding would render inactivation entirely absorbing. We reproduced this limiting case by eliminating all return pathways from inactivated states (Eq. 2: $\mathrm{k}_{1}, \mathrm{k}_{3} \rightarrow 0$ ). While this model of lidocaine action enhanced the decay rate of FQ currents to some degree (Fig. 9; bottom, middle), the acceleration of decay was not adequate to simulate lidocaine effects at concentrations $\geq 100 \mu \mathrm{M}$.
While Kuo and Bean (16) treated diphenylhydantoin binding and inactivation as independent and competitive processes, our data (Figs. 1-3) suggest that lidocaine actually restores and enhances the impaired FQ inactivation mechanism. The inability of FQ channels to inactivate normally results partly from destabilization of the inactivated state of the channel, such that inactivation is no longer absorbing (35); in the hinged-lid model of fast inactivation $(13,59)$, this effect of FQ would be interpreted as a defect in the binding or latching mechanism that holds the gate shut. However, the substantial increase in mean open time for FQ relative to wild-type (Fig. $4 C$ ) indicates that the association rate of the inactivation gate with the open channel is also reduced (31). In studies of cloned human cardiac Na channels (35), the analogous III-IV linker mutations also inhibited inactivation from closed states. Hence, if lidocaine restores and stabilizes the fast inactivation mechanism, it must increase the inactivation-gate association (forward) rates from both the closed and open states $\left(k_{2}\right.$ and $k_{4}$, respectively), and at the same time augment inactivated-state stability by decreasing the inactivation gate dissociation (reverse) rates $\left(\mathrm{k}_{1}\right.$ and $\left.\mathrm{k}_{3}\right)$. A drug-induced reduction in free en$\operatorname{ergy}(\Delta G)$ for the inactivated channel conformation would produce these kinetic effects. This idea is depicted in the simplified free energy diagram shown below.

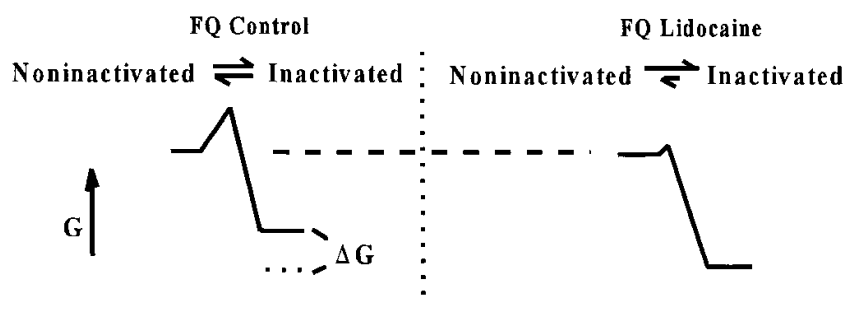

To quantitatively examine this paradigm for drug action, we assumed that drug affinity for the inactivated states (bottom) exceeded that for the noninactivated states (top) in Eq. 2. This assumption allows the rate constants controlling inactivation of drug-associated states to differ from those controlling drug-free gating without violating thermodynamic principles. To implement this effect using the model shown in Eq. 2, as a first approximation we used scale factors to render dosedependent changes in the inactivation forward and reverse rate constants constants as follows:

$* \mathrm{k}_{2}=\mathrm{k}_{2}\left(1+\mathrm{A}_{\text {on }}\right.$ [Lidocaine] $) ; \quad * \mathrm{k}_{4}=\mathrm{k}_{4}\left(1+\mathrm{A}_{\text {on }}\right.$ [Lidocaine $)$

$* \mathrm{k}_{1}=\mathrm{k}_{1}\left(1-\mathrm{A}_{\text {off }}[\right.$ Lidocaine $\left.]\right) ; \quad * \mathrm{k}_{3}=\mathrm{k}_{3}\left(1-\mathrm{A}_{\text {off }}[\right.$ Lidocaine $\left.]\right)$

In Eq. 3b, the influence of lidocaine on the free energy of inactivation gate association and dissociation is embedded in the scale factors $A_{\text {on }}$ and $A_{\text {off }}$ respectively. Fig. 9 (Model $C$, right) shows that this model for lidocaine action faithfully reproduced the dose-dependent effects of lidocaine on FQ currents (compare with Fig. $1 A$ ). When the inactivation rate constants $\left(\mathrm{k}_{1}, \mathrm{k}_{2}, \mathrm{k}_{3}\right.$, and $\left.\mathrm{k}_{4}\right)$ were modified by drug using $\mathrm{A}_{\mathrm{on}}=$ $9.85 \times 10^{-3}$ and $A_{\text {off }}=9.9 \times 10^{-4}$, the predicted drug-induced change in FQ mean open time was $7 \%$ at $100 \mu \mathrm{M}(1.61 \mathrm{~ms})$, and $15 \%$ at $300 \mu \mathrm{M}(1.44 \mathrm{~ms})$. These small changes in open time are entirely consistent with our open-time measurements in cell-patches (Fig. 5 A), where lidocaine $(300 \mu \mathrm{M})$ caused a small, but statistically insignificant, reduction in mean open time at membrane potentials $\geq-30 \mathrm{mV}$. 


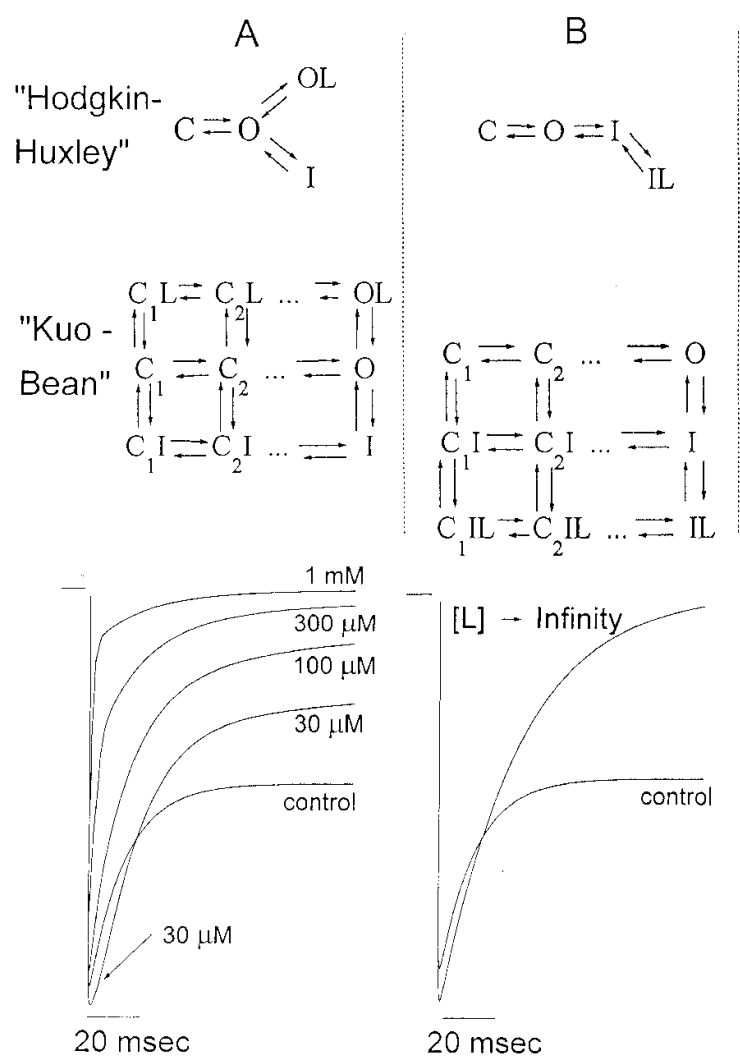

Through lowering the reverse inactivation rate constants $\left(\mathrm{k}_{1}, \mathrm{k}_{3}\right)$ and increasing the forward inactivation rate constants $\left(\mathrm{k}_{2}, \mathrm{k}_{4}\right)$, lidocaine altered the allosteric coupling relationship between activation and inactivation, causing channels to inactivate more readily from closed states in the activation sequence, as well as from the open state. For Model C, the probability that a channel reaching state $\mathrm{C}_{4}$ (Eq. 2) will inactivate directly to $\mathrm{C}_{4} \mathrm{I}$ is only 0.04 in the absence of drug, but increases to 0.15 in $300 \mu \mathrm{M}$ lidocaine. Alteration in activation-inactivation coupling may also underlie the difference in lidocaine action between FQ and QQQ (Fig. 2, $B$ and $C$ ). Nearly $75 \%$ of wild-type channels inactivate from closed states (rather than open) during a depolarization to $-20 \mathrm{mV}(31,35)$, causing null sweeps in cell-attached patch recordings. In our model, the FQ rate constants for inactivation from early closed states (Eq. 2, state $\left.\mathrm{C}_{1}\right)$ were markedly enhanced over wild-type $\left(\mathrm{k}_{2}\right.$ increased 100 -fold over wild-type, see table in Fig. 8). While a recent single-channel analysis of FQ and QQQ-hH1 channels suggested that closed state inactivation was rare for these mutants (35), the lack of null FQ and QQQ sweeps relative to wild-type may only imply that FQ and QQQ inactivated states are less absorbing. Consistent with this idea, the FQ model return rate constant (Eq. 2, $\mathrm{k}_{1}$ ) from the earliest closed-inactivated state $\left(\mathrm{CI}_{1}\right)$ was exceptionally large (Fig. $8, \sim 10^{6} \mathrm{~s}^{-1}$ ) compared to wild-type. Assuming an even greater effect on QQQ closed state inactivation forward and reverse rate constants, a substantial number of QQQ openings during the initial depolarization period may derive from channels that briefly transit through closed inactivated states before opening, and therefore contribute to the peak whole-cell current. If lidocaine speeds entry into the closed inactivated states and also enhances their absorbing character (as Model C suggests), then a
C
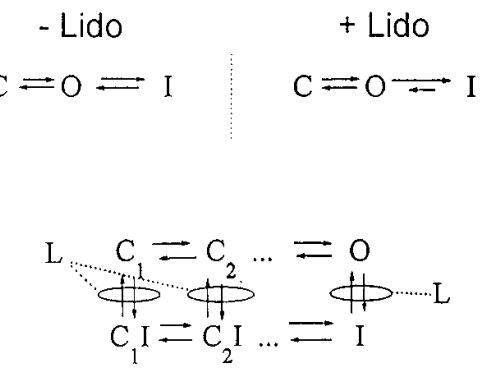

Figure 9. Comparison of three gating models for lidocaine effects on inactivation-disabled $\mathrm{Na}$ channels (see text). Models A and $\mathrm{B}$ are traditional modulatedreceptor models for high-affinity open and inactivated-channel block, respectively. In model $\mathrm{C}$, lidocaine possesses enhanced affinity for the inactivated channel, and also functions as an effector of allosteric gating by altering coupling between activation and inactivation. Only model C was capable of accurately simulating FQ macroscopic currents (see Fig. $1 A$ ) over the full range of lidocaine concentrations tested.

greater effect of lidocaine on the QQQ peak current might be anticipated. Such a mechanism would predict a delayed component in the first latency distribution for QQQ channels.

\section{Discussion}

Lidocaine restores inactivation of FQ channels. Site-directed mutagenesis of hydrophobic residues in the III-IV linker of the $\mathrm{Na}$ channel $\alpha$ subunit produces channels with impaired fast inactivation (13). Whole-cell currents from FQ channels exhibited slow decay, with $\sim 50 \%$ reduction from the peak after 100 $\mathrm{ms}$ at $-20 \mathrm{mV}$. Lidocaine hastened the decay of FQ current in both whole-cell recordings (Fig. 1) and ensemble-average currents from cell-attached patches (Fig. 4). Further, lidocaine restored use dependence to FQ channels by delaying recovery from inactivation (Fig. 3), apparently restoring wild-type inactivation gating characteristics. The $\mathrm{IC}_{50}$ for lidocaine block of FQ plateau current $(74 \mu \mathrm{M})$ approached the lidocaine $K_{\mathrm{d}}$ values recently reported for steady state block of wild-type channels fully inactivated by long depolarizing prepulses $(12 \mu \mathrm{M})$ (46), and differed markedly from the low $(\sim \mathrm{mM})$ affinities reported for open-pore lidocaine block (47).

It is well established that the rate of decay of wild-type whole-cell $\mathrm{Na}$ current is delayed by a prominent bursting mode, and that saturating coexpression of $\beta_{1}$ subunit in Xenopus oocytes essentially eliminates this gating mode (36-39) and accelerates the decay of whole-cell current $(23,24)$. The presence of an analogous bursting mode in FQ single channel records without $\beta_{1}$ coexpression (31) prompted us to evaluate whether lidocaine may act selectively on such channels, and thus enhance the decay of ensemble average FQ current. The prominent acceleration of FQ whole-cell current decay by 
lidocaine (Fig. 2) even with $\beta_{1}$ subunit coexpression (which essentially eliminates bursting openings [31]) suggests that lidocaine must accelerate FQ current decay primarily through an effect on channels gating in the predominant, nonbursting mode. We did observe a striking rightward shift in the FQlidocaine dose response curve with $\beta_{1}$ coexpression. This $\beta_{1}$ induced shift in the FQ dose-response curve is nearly identical to that reported recently for lidocaine block of human heart (hH1) wild-type Na channels (40). The basis of this shift is poorly understood, but may reflect an effect of $\beta_{1}$ on the lidocaine binding site (40).

The effect of lidocaine on FQ current decay could result from drug-induced stabilization or acceleration of inactivation; alternatively, accelerated decay of whole-cell current could be due to lidocaine block of the open pore in a manner analogous to block of delayed rectifier potassium currents by tetraethylammonium ion derivatives (41). This uncertainty reflects longstanding controversy over the relative importance of the openstate and the inactivated-state in local anesthetic-induced use dependence. The decay acceleration seen with FQ was markedly attenuated in QQQ, a mutation with more defective inactivation (Fig. 2 E). Given the similarity in FQ and QQQ single channel conductance (35), it is unlikely that the increased sensitivity of the QQQ peak whole cell current to lidocaine (and the relative sparing of FQ and wild-type peak current) results from enhanced sensitivity of the open QQQ channel to drug. Rather, we suggest that the more severe inactivation lesion may allow some channels to inactivate transiently from closed states and then open early during depolarization, thereby increasing the lidocaine sensitivity of peak whole-cell QQQ current.

To examine more directly the effects of local anesthetics on the open pore, single FQ channels were examined. A lowaffinity voltage-dependent reduction in unitary current amplitude was identified for both lidocaine (Fig. 5 B) and QX-314 (Fig. $6 \mathrm{~B}$ ), consistent with a rapid flicker-block mechanism, with an estimated electrical distance to the binding site from the cytoplasmic side $(\delta=0.5-0.7)$. This electrical distance is similar to that measured in recent work with single cardiac $\mathrm{Na}$ channels and permanently charged lidocaine derivatives $(\delta=$ 0.70) (8). The rapid kinetics of this block process would only scale the ensemble average current; further, both lidocaine (Fig. 4 B) and QX-314 (Fig. 7 D) enhance macroscopic inactivation at concentrations below the $K_{\mathrm{d}}$ 's for flicker block, suggesting that these drug effects are mechanistically distinct. While these experiments cannot discern the relative importance of the charged and uncharged local anesthetic species, the charged congener is certainly capable of enhancing inactivation in FQ channels.

A slower mode of open-channel block may appear as a reduction in mean open time in single-channel experiments. We detected a small but statistically insignificant change in FQ mean open time in cell-attached patches using lidocaine concentrations that markedly reduced the number of reopenings during a depolarizing clamp step (Fig. 4). Although these results differ from the reduction in mean open time by lidocaine originally reported by Nilius for $\mathrm{Na}$ channels in guinea pig ventricular myocytes (63), our results are nonetheless consistent with those of Grant et al. (64) in rabbit ventricular myocytes, as well as recent studies of inactivation-disabled human cardiac $\mathrm{Na}$ channels and lidocaine (14). The invariance of the mean open time would suggest that binding to the open channel with association kinetics similar to the wild-type fast-inacti- vation process does not occur. In a setting of coexistent openchannel flicker block that is rapid relative to intrinsic gating, measured open times may be prolonged by an amount that depends on the drug binding kinetics (65). This effect could mask a discrete lidocaine-induced shortening of the open-state dwell time. However, lidocaine accelerated the decay of macroscopic FQ current at doses well below $100 \mu \mathrm{M}$ (Fig. 1); in contrast, the $K_{\mathrm{d}}$ for lidocaine rapid block of the open pore was $877 \mu \mathrm{M}$ (Fig. 5 B). Hence, rapid open-channel block would have little effect on the measured open time at low lidocaine doses that are sufficient to accelerate FQ current decay. Nonetheless, on the basis of these single-channel experiments we cannot exclude the possibility that drug associates with the open channel very slowly, as the associated change in mean open time would undoubtedly fall below our detection threshold. Open-channel block on this time scale (seconds) may scale the FQ macroscopic current, but should not restore wild-type decay during a $100 \mathrm{~ms}$ depolarizing pulse.

$A$ revised model for local anesthetic action. Using a contemporary model of Na channel gating (Eq. 2) which considers fast inactivation as a particle-binding process allosterically coupled to activation (16), we simulated the effects of lidocaine on FQ channels using modulated-receptor models of open or inactivated state high-affinity binding (Fig. 9, $A$ and $B$ ). When constrained by the single channel data from cell-attached patches, we found both models to be inadequate to reproduce the effects of lidocaine on FQ currents over a range of concentrations. The inability of the modulated receptor models to reproduce the experimental data challenges the traditional paradigm for local anesthetic action which relies heavily on the formation of nonconducting drug-associated open or inactivated states. The notion of a drug-induced reduction in the free energy for inactivation (Fig. $9 \mathrm{C}$ ) enabled us to simulate the effects of lidocaine on FQ current using only two additional parameters $\left(A_{o n}, A_{o f f}\right)$. As an effector of allosteric coupling, this paradigm for local anesthetic action bears general similarity to a recently proposed mechanism for dihydropyridine agonist action on the L-type Ca channel (60).

Many sites in the Na channel influence binding of the inactivation particle (the III-IV linker) to the intracellular face of the pore. On the III-IV linker itself, in addition to the hydrophobic IFM triplet, deletion of nearby residues (KPQ) disrupts cardiac Na channel inactivation (66). In addition, mutation of particular residues on the intracellular ends of transmembrane segments S4-S6 on domains III and IV attenuate fast inactivation, perhaps through disrupting the receptor or latch for the inactivation gate (67-70). The effects of these mutations on local anesthetic block are varied. The paramyotonia mutation T1313M on the III-IV linker reduced use-dependent block of skeletal muscle $\mathrm{Na}$ channels by lidocaine (71). However, low doses of the lidocaine derivative mexilitine blocked the noninactivating plateau current generated by intracellular S4/S5 mutations that disrupt the putative inactivation gate receptor (68). When considered in light of our data, a compelling hypothesis is that lidocaine, when bound to the channel, strengthens the latch mechanism. In this manner, drug would similarly enhance inactivation of wild-type and inactivation-disabled channels.

While binding in the pore may underlie low-affinity voltage-dependent block by charged local anesthetics, the higheraffinity binding site that influences $\mathrm{Na}$ channel inactivation remains uncertain. However, our results showing high-affinity 
block of channels in excised inside-out patches by QX-314 (Fig. 7) suggests an intracellular locus. Although mutation of the IV-S6 phenylalanine (F1764A) attenuated use-dependent block by etidocaine in rat brain type IIA Na channels (11), this mutation also disrupted inactivation gating (67). Such an effect on inactivation can indirectly influence local anesthetic action; hence, the reduction in use-dependent block by this mutation may not specifically define the location of the local anesthetic receptor. The profound influence of externally positioned $\mathrm{mu}-$ tations on fast inactivation $(30,52,72)$ suggests that the structural characteristics of the inactivation-gate docking site are sensitive to remote allosteric effects. Recent work suggests that the externally bound $\alpha$-scorpion and sea anemone toxins (44) disrupt fast inactivation through a conformational change transmitted through the S3 and S4 transmembrane repeats (73). Additionally, the externally positioned IV-S4 paramyotonia mutation R1448C, which uncouples activation from fast inactivation (52), renders skeletal muscle Na channels more sensitive to lidocaine block (71). Hence, inactivation is sensitive to induced conformational changes that modify coupling between activation and inactivation, and lidocaine may enhance inactivation as an effector of this allosteric gating mechanism. While it is possible that alternative models may fit our data equally well, these studies suggest that a contemporary theory of local anesthetic action should incorporate coupling between gating process. Although nontraditional as a model for local anesthetic action, an analogous indirect mechanism has been proposed for $\mathrm{Ca}^{2+}$-sensitive inactivation in L-type Ca channels, where the divalent cations bind to an EF-hand motif and secondarily inhibit channel opening through an induced conformational change (74).

\section{Acknowledgments}

We wish to thank Dr. Gary Yellen for his comments on the Markov modeling.

This research was supported by National Institutes of Health (NIH) grant R01 HL52768 (E. Marban). Salary support was provided by NIH grants K11 HL02639 (J.H. Lawrence) and R01 HL50411 (G.F. Tomaselli); a fellowship from the American Heart Association (AHA), Maryland Affiliate (H.B. Nuss); the AHA-Clinician Scientist Award and the Passano Clinician Scientist Award of the Johns Hopkins School of Medicine (J.R. Balser), and a grant from the Whitaker Foundation (J.H. Lawrence).

\section{References}

1. Courtney, K.R. 1975. Mechanism of frequency-dependent inhibition of sodium currents in the frog myelinated nerve by the lidocaine derivative gea 968. J. Pharmacol. Exp. Ther. 195:225-236.

2. Hille, B. 1977. Local anesthetics: hydrophilic and hydrophobic pathways for the drug-receptor reaction. J. Gen. Physiol. 69:497-515.

3. Hondeghem, L.M., and B.G. Katzung. 1977. Time- and voltage-dependent interactions of the antiarrhythmic drugs with cardiac sodium channels. Biochim. Biophys. Acta. 472:373-398.

4. Matsubara, T., C. Clarkson, and L. Hondeghem. 1987. Lidocaine blocks open and inactivated cardiac sodium channels. Naunyn-Schmiedeberg's Archives of Pharmacology. 336:224-231.

5. Strichartz, G.R. 1973. The inhibition of sodium currents in myelinated nerve by quarternary derivatives of lidocaine. J. Gen. Physiol. 62:37-57.

6. Cahalan, M.D. 1978. Local anesthetic block of sodium channels in normal and pronase-treated squid axons. Biophys. J. 23:285-311.

7. Yeh, J.Z. 1978. Sodium inactivation mechanism modulates qx-314 block of sodium channels. Biophys. J. 24:569-574.

8. Gingrich, K.J., D. Beardsley, and D.T. Yue. 1993. Ultra-deep blockade of $\mathrm{Na}$ channels by a quaternary ammonium ion: catalysis by a transition-intermediate state? J. Physiol. 471:319-341.
9. Bean, B.P., C.J. Cohen, and R.W. Tsien. 1983. Lidocaine block of cardiac sodium channels. J. Gen. Physiol. 81:613-642.

10. Schwarz, W., P.T. Palade, and B. Hille. 1977. Local anesthetic effect of $\mathrm{pH}$ on use-dependent block of sodium channels in frog muscle. Biophys. J. 20: 343-368.

11. Ragsdale, D.S., J.C. McPhee, T. Scheuer, and W.A. Catterall. 1994. Molecular determinants of state-dependent block of $\mathrm{Na}^{+}$channels by local anesthetics. Science (Wash. DC). 265:1724-1728.

12. Cahalan, M.D., and W. Almers. 1979. Interactions between quaternary lidocaine, the sodium channel gates, and tetrodotoxin. Biophys. J. 27:39-56.

13. West, J., D. Patton, T. Scheuer, Y. Wang, A.L. Goldin, and W.A. Catterall. 1992. A cluster of hydrophobic amino acid residues required for fast $\mathrm{Na}^{+}$-channel inactivation. Proc. Natl. Acad. Sci. USA. 89:10910-10914.

14. Bennett, P.B., C. Valenzuela, C. Li-Qiong, and R.G. Kallen. 1995. On the molecular nature of the lidocaine receptor of cardiac $\mathrm{Na}^{+}$channels. Circ. Res. 77:584-592.

15. O’Leary, M.E., R.G. Kallen, and R. Horn. 1994. Evidence for a direct interaction between internal tetra-alkylammonium cations and the inactivation gate of cardiac sodium channels. J. Gen. Physiol. 104:523-539.

16. Kuo, C.C., and B.P. Bean. 1994. $\mathrm{Na}^{+}$channels must deactivate to recover from inactivation. Neuron. 12:819-829.

17. Trimmer, J.S., S.S. Cooperman, S.A. Tomiko, J. Zhou, S.M. Crean, M.B. Boyle, R.G. Kallen, Z. Sheng, R.L. Barchi, F.J. Sigworth et al. 1989. Primary structure and functional expression of a mammalian skeletal muscle sodium channel. Neuron. 3:33-49.

18. Kunkel, T.A. 1985. Rapid and efficient site-specific mutagenesis without phenotypic selection. Proc. Natl. Acad. Sci. USA. 82:488-492.

19. Backx, P.H., D.T. Yue, J.H. Lawrence, E. Marban, and G.F. Tomaselli. 1992. Molecular localization of an ion-binding site within the pore of mammalian sodium channels. Science (Wash. DC). 257:248-251.

20. Kunkel, T.A., J.D. Roberts, and R.A. Zakour. 1987. Rapid and efficien site-specific mutagenesis without phenotypic selection. Methods Enzymol. 154: 367-383.

21. Krieg, P.A., and D.A. Melton. 1984. Functional messenger RNAs are produced by SP6 in vitro transcription of cloned cDNAs. Nucleic Acids Res. 12: $7057-7070$.

22. Sanger, F., S. Nicklen, and A.R. Coulson. 1977. DNA sequencing with chain-terminating inhibitors. Proc. Natl. Acad. Sci. USA. 74:5463-5467.

23. Isom, L.L., K.S. Dejongh, D.E. Patton, B.F.X. Reber, J. Offord, H Charbonneau, K. Walsh, A.L. Goldin, and W.A. Catterall. 1992. Primary structure and functional expression of the beta-1 subunit of the rat brain sodium channel. Science (Wash. DC). 256:839-842.

24. Cannon, S.C., A.I. McClatchey, and J.F. Gusella. 1993. Modification of the $\mathrm{Na}^{+}$current conducted by the rat skeletal muscle $\alpha$ subunit by coexpression with a human brain $\beta$ subunit. Pflïgers Arch. 423:155-157.

25. Sambrook, J., E.F. Fritsch, and T. Maniatis. 1989. Molecular Cloning: A Laboratory Manual. Cold Spring Harbor Laboratory Press, Cold Spring Harbor, NY. 3.19 pp.

26. Hamill, O.P., A. Marty, E. Neher, B. Sakmann, and F.J. Sigworth. 1981. Improved patch-clamp techniques for high-resolution current recording form cells and cell-free membrane patches. Pflügers Arch. 391:85-100.

27. Nuss, H.B., N. Chiamvimonvat, M.T. Perez-Garcia, G.F. Tomaselli, and E. Marban. 1995. Functional association of the $\beta_{1}$ subunit with human cardiac (hH1) and rat skeletal muscle $(\mu 1)$ sodium channel $\alpha$ subunits expressed in $X e$ nopus oocytes. J. Gen. Physiol. 106:1171-1191.

28. Colquhoun, D., and F.J. Sigworth. 1983. Fitting and statistical analysis of single-channel records. In Single-Channel Recording. B. Sakmann and E. Neher, editors. Plenum Press, New York. 191-263.

29. Balser, J.R., D.M. Roden, and P.B. Bennett. 1990. Global parameter optimization for cardiac potassium channel gating models. Biophys. J. 57:433-444.

30. Tomaselli, G.F., N. Chiamvimonvat, H.B. Nuss, J.R. Balser, M.T. PerezGarcia, R.H. Xu, D.W. Orias, P.H. Backx, and E. Marban. 1995. A mutation in the pore of the sodium channel alters gating. Biophys. J. 68:1814-1827.

31. Lawrence, J.H., D.W. Orias, J.R. Balser, H.B. Nuss, G.F. Tomaselli, and E. Marban. 1996. Single-channel analysis of inactivation-defective rat skeletal muscle sodium channels containing the F1304Q mutation. Biophys. J. 71:12851294.

32. Hindmarsh, A.C. 1983. Odepack, a systematized collection of ode solvers. In Scientific Computing. R.S. Stepleman, editor. North-Holland Company, Amsterdam. 55-64.

33. Petzold, L.R. 1983. Automatic selection of methods for solving stiff and nonstiff systems of ordinary differential equations. Society for Industrial and Applied Mathematics Journal on Scientific and Statistical Computing 4:136-148.

34. Nelder, J.A., and R. Mead. 1965. A Simplex method for function minimization. Computer Journal. 7:308-313.

35. Hartmann, H.A., A.A. Tiedeman, S.F. Chen, A.M. Brown, and G.E. Kirsch. 1994. Effects of III-IV linker mutations on human heart $\mathrm{Na}^{+}$channel inactivation gating. Circ. Res. 75:114-122.

36. Zhou, J., J.F. Potts, J.S. Trimmer, W.S. Agnew, and F.J. Sigworth. 1991. Multiple gating modes and the effect of modulating factors on the $\mu 1$ sodium channel. Neuron. 7:775-785.

37. Moorman, J.R., G.E. Kirsch, A.M.J. VanDongen, R.H. Joho, and A.M. 
Brown. 1990. Fast and slow gating of sodium channels encoded by a single mRNA. Neuron. 4:243-252.

38. Bennett, P.B., N. Makita, and A.L. George. 1993. A molecular basis for gating mode transitions in human skeletal muscle sodium channels. FEBS Lett. 326:21-24.

39. Chang, S.Y., J. Satin, and H.A. Fozzard. 1996. Modal behavior of the $\mu$ l $\mathrm{Na}^{+}$channel and effects of coexpression of the $\beta_{1}$ subunit. Biophys. J. 70:25812592.

40. Makielski, J.C., J.T. Limberis, S.Y. Chang, Z. Fan, and J.W. Kyle. 1996. Coexpression of $\beta_{1}$ with cardiac sodium channel $\alpha$ subunits in oocytes decreases lidocaine block. Molecular Pharmacology. 49:30-39.

41. Armstrong, C.M. 1971. Interaction of tetraethylammonium ion derivatives with the potassium channels of giant axons. J. Gen. Physiol. 58:413-437.

42. Nuss, H.B., J.R. Balser, D.W. Orias, J.H. Lawrence, G.F. Tomaselli, and E. Marban. 1996. Coupling between fast and slow inactivation revealed by analysis of a point mutation (F1304Q) in $\mu 1$ rat skeletal muscle sodium channels. $J$. Physiol. 494:411-429.

43. Balser, J R , H B. Nuss, DN. Romashko, E. Marban, and G.F. Tomaselli. 1996. Functional consequences of lidocaine binding to slow-inactivated sodium channels. J. Gen. Physiol. 107:643-658.

44. Hille, B. 1992. Ionic channels of excitable membranes. Sinauer Associates, Inc. Sunderland, MA.

45. Woodhull, A.M. 1973. Ionic blockage of sodium channels in nerve. $J$. Gen. Physiol. 61:687-708.

46. Nuss, H.B., G.F. Tomaselli, and E. Marban. 1995. Cardiac sodium channels (hH1) are intrinsically more sensitive to tonic block by lidocaine than are skeletal muscle ( $\mu 1)$ channels. J. Gen. Physiol. 106:1193-1210.

47. Zamponi, G.W., D.D. Doyle, and R.J. French. 1993. Fast lidocaine block of cardiac and skeletal muscle sodium channels: one site with two routes of access. Biophys. J. 65:80-90.

48. Hodgkin, A.L. and A.F. Huxley. 1952. A quantitative description of membrane current and its application to conduction and excitation in nerve. $J$. Physiol. 117:500-544.

49. Horn, R., J.B. Patlak, and C.F. Stevens. 1981. Sodium channels need not open before they inactivate. Nature (Lond.). 291:426-427.

50. Lawrence, J.H., D.T. Yue, W.C. Rose, and E. Marban. 1991. Sodium channel inactivation from resting states in guinea-pig ventricular myocytes. $J$. Physiol. 443:629-650.

51. Armstrong, C.M., and F. Bezanilla. 1977. Inactivation of the sodium channel. II. Gating current experiments. J. Gen. Physiol. 70:567-590.

52. Chahine, M., A.L. George, M. Zhou, S. Ji, W. Sun, R. Barchi, and R. Horn. 1994. Sodium channel mutations in paramyotonia congenita uncouple inactivation from activation. Neuron. 12:281-294.

53. Horn, R., and C.A. Vandenberg. 1984. Statistical properties of single sodium channels. J. Gen. Physiol. 84:505-534.

54. Patlak, J. 1991. Molecular kinetics of voltage-dependent $\mathrm{Na}^{+}$channels. Phys. Rev. 71:1047-1080.

55. Vassilev, P.M., T. Scheuer, and W.A. Catterall. 1988. Identification of an intracellular peptide segment involved in sodium channel inactivation. Science (Wash. DC). 241:1658-1661.

56. Vassilev, P.M., T. Scheuer, and W.A. Catterall. 1989. Inhibition of inactivation of single sodium channels by a site-directed antibody. Proc. Natl. Acad.
Sci. USA. 86:8147-8151.

57. Stühmer, W., F. Conti, H. Suzuki, X. Wang, M. Noda, N. Yahagi, H Kubo, and S. Numa. 1989. Structural parts involved in activation and inactivation of the sodium channel. Nature (Lond.). 339:597-603.

58. Moorman, J.R., G.E. Kirsch, A.M. Brown, and R.H. Joho. 1990 Changes in sodium channel gating produced by point mutations in a cytoplasmic linker. Science (Wash. DC). 250:688-691.

59. Patton, D.E., J.W. West, W.A. Catterall, and A.L. Goldin. 1992. Amino acid residues required for fast $\mathrm{Na}^{+}$-channel inactivation: charge neutralization and deletions in the III-IV linker. Proc. Natl. Acad. Sci. USA. 89:10905-10909.

60. Marks, T.N., and S.W. Jones. 1992. Calcium currents in the A7r5 smooth muscle-derived cell line: an allosteric model for calcium channel activation and dihydropyridine agonist action. J. Gen. Physiol. 99:367-390.

61. Monod, J., J. Wyman, and J.-P. Changeux. 1965. On the nature of allosteric transitions: a plausible model. J. Mol. Biol. 12:88-118.

62. Lawrence, J.H., D.W. Orias, H.B. Nuss, P.H. Backx, R.H. Xu, J.R. Balser, G.F. Tomaselli, and E. Marban. 1993. Retained access to the inactivated state in an inactivation-deficient mutant sodium channel. Circ. 88:I-185.

63. Nilius, B., K. Benndorf, and F. Markwardt. 1987. Effects of lidocaine on single cardiac sodium channels. J. Mol. Cell. Cardiol. 19:865-874.

64. Grant, A.O., M.A. Dietz, F.R. Gilliam III, and C.F. Starmer. 1989. Blockade of cardiac sodium channels by lidocaine: single-channel analysis. Circ Res. 65:1247-1262.

65. Neher, E., and J.H. Steinbach. 1978. Local anesthetics transiently block currents through single acetylcholine-receptor channels. J. Physiol. 277:153-176.

66. Bennett, P.B., K. Yazawa, M. Naomasa, and A.L. George. 1995. Molecular mechanism for an inherited cardiac arrhythmia. Nature (Lond.). 376:683-685.

67. McPhee, J.C., D.S. Ragsdale, T. Scheuer, and W.A. Catterall. 1995. A critical role for transmembrane segment IVS6 of the sodium channel $\alpha$ subunit in fast inactivation. J. Biol. Chem. 270:12025-12034.

68. Dumaine, R., Q. Wang, M.T. Keating, H.A. Hartmann, P.J. Schwartz, A.M. Brown, and G.E. Kirsch. 1996. Multiple mechanisms of $\mathrm{Na}^{+}$channellinked long-QT syndrome. Circ. Res. 78:916-924.

69. Depp, M.R., and A.L. Goldin. 1996. Probing S4-S5 regions of the rat brain sodium channel for the fast inactivation particle receptor site. Biophys. J. 70(Abstr.):317a.

70. McPhee, J.C., D.S. Ragsdale, T. Scheuer, and W.A. Catterall. 1996. A role for intracellular loop IVS4-S5 of the $\mathrm{Na}^{+}$channel $\alpha$ subunit in fast inactivation. Biophys. J. 70(Abstr.):318a.

71. Fan, Z., A.L. George, J.W. Kyle, and J.C. Makielski. 1996. Two paramyotonia congenita $\mathrm{Na}$ channel mutations induce an opposite affinity change for lidocaine. Biophys. J. 70 (Abstr.):319a.

72. Ji, S. A.L. George, R. Horn, and R.L. Barchi. 1996. Paramyotonia congenita mutations reveal different roles for segments S3 and S4 of domain D4 in hSkM1 sodium channel gating. J. Gen. Physiol. 107:183-194.

73. Rogers, J.C., Y. Ou, T. Scheuer, and W.A. Catterall. 1996. Molecula determinants of $\alpha$-scorpion and anemone toxin binding to the $\mathrm{Na}^{+}$channel Biophys. J. 70(Abstr.):319a.

74. de Leon, M., Y. Wang, L. Jones, E. Perez-Reyes, X. Wei, T.W. Soong, T.P. Snutch, and D.T. Yue. 1995. Essential $\mathrm{Ca}^{2+}$-binding motif for $\mathrm{Ca}^{2+}$-sensitive inactivation of L-type $\mathrm{Ca}^{2+}$ channels. Science (Wash. DC). 270:1502-1506. 